\title{
Restricted conformal invariance in QCD and its predictive power for virtual two-photon processes
}

\author{
D. Müller* \\ TH Division, CERN, 1211 Geneva 23, Switzerland \\ (Received 9 March 1998; published 22 July 1998)
}

\begin{abstract}
The conformal algebra provides powerful constraints, which guarantee that renormalized conformally covariant operators exist in the hypothetical conformal limit of the theory, where the $\beta$-function vanishes. Thus, in this limit also the conformally covariant operator product expansion on the light cone holds true. This operator product expansion has predictive power for two-photon processes in the generalized Bjorken region. Only the Wilson coefficients and the anomalous dimensions that are known from deep inelastic scattering are required for the prediction of all other two-photon processes in terms of the process-dependent off-diagonal expectation values of conformal operators. It is checked that the next-to-leading order calculations for the flavor nonsinglet meson transition form factors are consistent with the corrections to the corresponding Wilson coefficients in deep inelastic scattering. [S0556-2821(98)04215-5]

PACS number(s): 12.38.Bx, 13.60. $-\mathrm{r}$
\end{abstract}

\section{INTRODUCTION}

In a massless theory, conformal symmetry has the ability to provide powerful predictions for physical quantities. However, conformal symmetry is broken on the quantum level due to the renormalization. This breaking is controlled by the conformal Ward identities (CWI) of dilatation and special conformal symmetry. It is often possible to redefine the conformal representation, implying that in the physical sector of the theory the conformal symmetry is only broken by the renormalization of the running coupling constant, which provides a symmetry-breaking term proportional to the $\beta$-function. If there exists a non-trivial (non-perturbative) fixed point such that the $\beta$-function vanishes, conformal invariance holds true. This is the so-called hypothetical conformal limit of the theory.

For instance, the Crewther relation [1] (also derived in [2]) is based on conformal invariance. The value of the Bjorken sum rule $K$ and the isovector part $R^{\prime}$ of the crosssection ratio $\sigma\left(e^{+} e^{-} \rightarrow\right.$ hadrons $) / \sigma\left(e^{+} e^{-} \rightarrow \mu^{+} \mu^{-}\right)$are computed in QCD up to the order $\alpha_{s}^{3}[3,4]$. Assuming conformal invariance for the axial vector-vector-vector (AVV) correlator, Crewther proved that the relation $3 S=K R^{\prime}$ holds true; $S$ is the anomalous constant, which is, corresponding to the Adler-Bardeen theorem, given by its one-loop value. Since the vector current and the flavor non-singlet axialvector current are conserved, the CWI for the AVV correlator tells us that conformal invariance can be broken only by the $\beta$-function [5]. This is actually the case for the available $\alpha_{s}^{3}$ order [6] (see also [7,8]).

It has been known for a long time that the conformal symmetry provides powerful constraints for the Wilson coefficients of the operator product expansion (OPE) for two local currents. This provides an improved OPE, which was called conformally covariant OPE and was studied in a num-

\footnotetext{
* On leave from the Institut für Theoretische Physik, Universität Leipzig, 04109 Leipzig, Germany.
}

ber of papers, starting in the 1970's with the work of Ferrara, Gatto and Grillo [9-13]. Employing the conformal Ward identities for the Green functions of composite operators, the conformal symmetry breaking in the interacting scalar theory was studied by Ferrara, Grillo and Parisi [14]. Making a non-trivial assumption about the form of the conformal Ward identities, the authors found that for a non-trivial fixed-point of the $\beta$-function the conformally covariant OPE holds true if the original scale dimensions, given by the canonical dimensions, are shifted by the anomalous dimensions.

Brodsky, Frishman, Lepage and Sachradja employed the conformally covariant expansion for a non-local operator, appearing in the definition of the pion distribution amplitude, to predict the evolution of this amplitude correctly at leading order (LO) [15] (see also [13]). Note that this requires a generalization of the OPE and an assumption about the conformal properties of this non-local operator. With this application a puzzle of the conformal invariance in gauge field theories sets up beyond the leading order. The next-toleading order (NLO) prediction for the eigenfunctions of the pion evolution kernel in the conformal limit contradicts the NLO calculation of the non-singlet evolution kernel in the modified minimal subtraction $(\overline{\mathrm{MS}})$ scheme [16-19]. Brodsky, Damgaard, Frishman and Lepage have shown that this breakdown of conformal symmetry is renormalizationscheme-dependent and that, in the special case of the $\varphi_{(6)}^{3}$-theory in six dimensions, conformal symmetry is preserved in the Pauli-Villars regularization at the considered order [20]. However, in QCD the conformal symmetry predictions could not be restored by a renormalization group transformation. Later, the breaking of conformal covariance for local conformal composite operators, appearing in the expansion of the considered non-local operator, was analyzed by the CWI and conformal constraints. It was found that the symmetry breaking in the $\overline{\mathrm{MS}}$ scheme (i) destroys the irreducible representation of the conformal algebra at leading order and (ii) that the conformal symmetry-breaking at this order provides the eigenfunctions of the computed evolution kernels in NLO [21,22], also for QCD. 
In this paper we do not reanalyze this issue in terms of a modified conformal OPE, which may be done in a straightforward manner by employing the CWI. Here we deal with the conformally covariant OPE for two local currents that have a well-known behavior under conformal transformations in the interacting theory. Neglecting the conformal symmetry-breaking terms that are proportional to the $\beta$-function allows us to give a first application to QCD processes.

The paper is organized as follows. For the convenience of the reader, in Sec. II we shortly review the conformal algebra and the irreducible representations that are needed, as well as the derivation of the conformally covariant OPE. Using conformal constraints, coming from the conformal algebra and the CWI, it will be proved in Sec. III that there exists a renormalization scheme in which the conformal covariance of composite operators holds true in the conformal limit. This allows the construction of the conformally covariant OPE in the interacting theory. In Sec. IV we employ this conformally covariant OPE to predict the scattering amplitude for two-photon processes in the light-cone-dominated region [23] covering the kinematics of deeply virtual Compton scattering (DVCS), which has recently been proposed to open a new window for the exploration of the nucleon contents [24-26]. Included are two well-known special cases, which have been measured: deep inelastic scattering (DIS) and one-meson production at large momentum transfer in two-photon collision [27-30]. In Sec. V we show that the existing NLO corrections for the flavor non-singlet meson transition form factors, computed in the $\overline{\mathrm{MS}}$ scheme, coincide with the prediction of the conformally covariant OPE. Finally, the knowledge of the higher-order corrections to the Bjorken sum rule allows us to point out phenomenological consequences for the pion transition form factor.

\section{CONFORMAL ALGEBRA AND THE CONFORMALLY COVARIANT OPE}

\section{A. Field theoretical conformal representations}

The conformal group is the maximal extension of the Poincare group that leaves the light cone invariant; it is thus of physical interest for light-cone-dominated as well as for high-energy processes. Beside the well-known Poincaré transformations, the conformal group consists of dilatations: $x^{\alpha} \rightarrow \rho x^{\alpha}$ and special conformal transformations: $x^{\alpha} \rightarrow\left(x^{\alpha}\right.$ $\left.+c^{\alpha} x^{2}\right) /\left(1+2 c x+c^{2} x^{2}\right)$. The latter is composed of an inversion $x^{\alpha} \rightarrow x^{\alpha} / x^{2}$, a translation $x^{\alpha} \rightarrow x^{\alpha}+c^{\alpha}$, and a further inversion. The conformal factor $1 /\left(1+2 c x+c^{2} x^{2}\right)$ is singular on the cone and so the special conformal transformations are not well defined as global transformations in the Minkowski space. Moreover, it is possible to transform noncausal connected regions into one another, which violates the principle of causality. To apply the conformal group to the quantum field theory in Minkowski space, it is sufficient in future studies to restrict ourselves to infinitesimal special conformal transformations: this avoids both of the mentioned problems. In the following we consider field theories (of polynomial form) with space-time dimensions larger than 2 , which are conformally invariant on the classical level.
For space-time dimension $n$, the algebra of the conformal group is isomorphic to the algebra $\operatorname{SO}(n, 2)$ and consists, besides of the algebra of the Poincare generators $M_{\alpha \beta}$ and $P_{\alpha}$ :

$$
\begin{gathered}
{\left[M_{\alpha \beta}, M_{\gamma \delta}\right]=i\left(-g_{\alpha \gamma} M_{\beta \delta}+g_{\alpha \delta} M_{\beta \gamma}+g_{\beta \gamma} M_{\alpha \delta}-g_{\beta \delta} M_{\alpha \gamma}\right),} \\
(2.1)
\end{gathered}
$$

of the following commutation relations for the dilatation generator $D$ and for the generator $K_{\alpha}$ of special conformal transformations:

$$
\begin{aligned}
{\left[D, K_{\alpha}\right] } & =i K_{\alpha}, \\
{\left[K_{\alpha}, P_{\beta}\right] } & =-2 i\left(g_{\alpha \beta} D+M_{\alpha \beta}\right), \quad\left[K_{\alpha}, K_{\beta}\right]=0, \\
{\left[D, P_{\alpha}\right] } & =-i P_{\alpha}, \\
{\left[M_{\alpha \beta}, K_{\gamma}\right] } & =i\left(g_{\beta \gamma} K_{\alpha}-g_{\alpha \gamma} K_{\beta}\right), \quad\left[D, M_{\alpha \beta}\right]=0 .
\end{aligned}
$$

The field theoretical representations with finite components have been classified on the basis of the induced representation theory [31]. In the following we deal with irreducible representations of the conformal algebra, where the action of the special conformal generator $K_{\lambda}$ on a basis field $\phi(x)$ vanishes at the point $x^{\alpha}=0$ :

$$
\begin{gathered}
{\left[\phi(x), M_{\alpha \beta}\right]_{\mid x=0}=\Sigma_{\alpha \beta} \phi(0), \quad[\phi(x), D]_{\mid x=0}=i d_{\phi} \phi(0),} \\
{\left[\phi(x), K_{\alpha}\right]_{\mid x=0}=0 .}
\end{gathered}
$$

Here $\phi=\varphi, \psi, A^{\mu}$ may be a scalar field $\varphi$, a fermionic field $\psi$ or a gauge field $A^{\mu}$ with scale dimension $d_{\phi}$. Conformal invariance in the classical theory requires that the fields are massless and that the scale dimensions are equal to the canonical dimensions $d_{\phi}^{\text {can }}$ of the fields. The representation of the Lorentz group is:

$$
\begin{gathered}
\Sigma_{\alpha \beta} \varphi=0, \quad \Sigma_{\alpha \beta} \psi=\frac{i}{4}\left[\gamma_{\alpha}, \gamma_{\beta}\right] \psi, \\
\Sigma_{\alpha \beta} A_{\mu}=i\left(g_{\mu \alpha} A_{\beta}-g_{\mu \beta} A_{\alpha}\right) .
\end{gathered}
$$

The theory of induced representation now provides the action of the generators at an arbitrary space-time point:

$$
\begin{aligned}
{\left[\phi(x), P_{\alpha}\right] } & =i \partial_{\alpha} \phi(x), \\
{\left[\phi(x), M_{\alpha \beta}\right] } & =\left(i\left[x_{\alpha} \partial_{\beta}-x_{\beta} \partial_{\alpha}\right]+\Sigma_{\alpha \beta}\right) \phi(x), \\
{[\phi(x), D] } & =i\left(d_{\phi}+x \partial\right) \phi(x), \\
{\left[\phi(x), K_{\alpha}\right] } & =i\left(2 x_{\alpha}\left(d_{\phi}+x \partial\right)-2 i \Sigma_{\alpha \beta} x^{\beta}-x^{2} \partial_{\alpha}\right) \phi(x) .
\end{aligned}
$$

\section{B. Conformally covariant operators}

Parton distribution functions and hadron distribution amplitudes are defined as expectation values of composite lightray operators. Their moments are expressed in terms of local 
operators. In the following, the local conformal two-particle operators are considered. For these operators the conformal symmetry at tree level yields that the operators do not mix under renormalization at LO and so they are essential in solving the evolution equations in the non-forward case. For the leading twist operators it is sufficient to consider the collinear conformal algebra, which is isomorphic to $\mathrm{SU}(1,1) \equiv \mathrm{SO}(2,1)$. The four generators are obtained by projection onto the light cone:

$$
P_{+}=\tilde{n}_{\alpha} P^{\alpha}, \quad K_{-}=\tilde{n}_{\alpha}^{\star} K^{\alpha}, \quad D, \quad M_{-+}=\tilde{n}_{\alpha}^{\star} M^{\alpha \beta} \tilde{n}_{\beta},
$$

where $\tilde{n}, \tilde{n}^{\star}$ are light-cone vectors with the normalization $\tilde{n} \tilde{n}^{\star}=1$. They generate the projective transformation onto the line and satisfy the commutation relation [coming from the algebra (2.2)]:

$$
\begin{aligned}
{\left[M_{-+}, K_{-}\right] } & =\left[D, K_{-}\right]=i K_{-}, \\
{\left[K_{-}, P_{+}\right] } & =-2 i\left(D+M_{-+}\right), \\
{\left[M_{-+}, P_{+}\right] } & =\left[D, P_{+}\right]=-i P_{+}, \quad\left[M_{-+}, D\right]=0 .
\end{aligned}
$$

Acting with the step-up operator $P_{+}$on $\phi(0)$ generates an infinite-dimensional representation, the so-called conformal tower $\left\{\phi(0), P_{+} \phi(0), P_{+}^{2} \phi(0), \ldots\right\}$. The operator $K_{-}$acts as a step-down one, and annihilates the lowest member $\phi(0)$, i.e. $K_{-} \phi(0)=0$. As for $D$ and $M_{-+}$, they are diagonal operators, which give the scale dimension and the spin of the members, respectively. The spectrum of the Casimir operator

$$
C=\frac{1}{2} P_{+} K_{-}-\frac{1}{4}\left(D+M_{-+}\right)^{2}-\frac{i}{2}\left(D+M_{-+}\right)
$$

is $j(j+1)$, where $j$ is the conformal spin.

Conformal composite operators can be constructed by different methods [32-34], for instance by decomposition of the direct product of two towers $\left\{\phi_{i}(0), P_{+} \phi_{i}(0), \ldots\right\}$ into irreducible representations. The Clebsch-Gordan coefficients are given by the coefficients of the Jacobi polynomials $P_{k}^{\left(\nu_{1}-1 / 2, \nu_{2}-1 / 2\right)}$,

$$
\begin{aligned}
& O_{k l}\left(\nu_{1}, \nu_{2}\right)=\left(i \tilde{n} \partial_{+}\right)^{l} \phi_{1}(0) \Gamma \\
& \times P_{k}^{\left(\nu_{1}-1 / 2, \nu_{2}-1 / 2\right)}\left(\frac{\tilde{n} \partial_{-}}{\tilde{n} \partial_{+}}\right) \phi_{2}(0), \\
& l \geqslant k, \quad \nu_{i}=d_{\phi_{i}}+s_{\phi_{i}}-1 / 2
\end{aligned}
$$

where $\partial_{ \pm}^{\nu}=\vec{\partial}^{\nu} \pm \overleftarrow{\partial}^{\nu}$ and $\Gamma$ contains the spin and, eventually, further global group theoretical structures. To ensure gauge invariance in a given gauge field theory, the partial derivatives have to be replaced by the covariant ones, which does not spoil the conformal properties of the operator. The index $\nu_{i}$ is determined by both the scale dimension and the spin of the corresponding field $\phi_{i}$. The action of the collinear conformal generators are given by

$$
\begin{aligned}
& {\left[O_{k l}\left(\nu_{1}, \nu_{2}\right), P_{+}\right]=O_{k l+1}\left(\nu_{1}, \nu_{2}\right)} \\
& {\left[O_{k l}\left(\nu_{1}, \nu_{2}\right), K_{-}\right]=2(k-l)\left(k+l+\nu_{1}\right.} \\
& \left.+\nu_{2}\right) O_{k l-1}\left(\nu_{1}, \nu_{2}\right) \text {, } \\
& {\left[O_{k l}\left(\nu_{1}, \nu_{2}\right), D\right]=i\left(d_{1}+d_{2}+l\right) O_{k l}\left(\nu_{1}, \nu_{2}\right),}
\end{aligned}
$$

$$
\left[O_{k l}\left(\nu_{1}, \nu_{2}\right), M_{-+}\right]=i\left(s_{1}+s_{2}+l\right) O_{k l}\left(\nu_{1}, \nu_{2}\right),
$$

where $\left(d_{1}+d_{2}+l\right)$ and $\left(s_{1}+s_{2}+l\right)$ are the dimension and the spin, respectively, of the operator $O_{k l}\left(\nu_{1}, \nu_{2}\right)$. The lowest member in each tower is $O_{k k}\left(\nu_{1}, \nu_{2}\right)$.

In the following we often consider conformal operators in the $\varphi_{(6)}^{3}$ theory, where the field has the canonical dimension $d_{\varphi}=2$, and in QCD for the non-singlet channel, where the quark fields have $d_{\psi}=3 / 2$ and $s=1 / 2$, so that in both cases $\nu_{1}=\nu_{2}=3 / 2$. Since $\nu_{1}=\nu_{2}$, the Jacobi polynomials can be expressed by the Gegenbauer polynomials $C_{k}^{\nu}$ :

$$
P_{k}^{(\nu-1 / 2, \nu-1 / 2)}(x)=\frac{(\nu+1 / 2)_{k}}{(2 \nu)_{k}} C_{k}^{\nu}(x),
$$

where $(a)_{n}=\Gamma(a+n) / \Gamma(a)$ is the Pochhammer symbol.

\section{Conformally covariant OPE}

Here we refer to the work of Ferrara, Gatto and Grillo [9-11]. The construction of the conformally covariant OPE is based on the behavior of the conformal operators under infinitesimal conformal transformations, which is characterized by their scaling dimension and their conformal spin. To simplify the notation, only the scalar case will be considered. In Sec. III C the derivation reviewed here will be applied to the product of two electromagnetic currents. We restrict ourselves to leading twist and assume that the following symmetrized and traceless conformal operators with scale dimension $l_{k}+l-k=d_{1}+d_{2}+l$ form a complete basis:

$$
O_{\alpha_{1} \ldots \alpha_{l}, k}(x)=\operatorname{S}_{\{\alpha\}} i^{(l-k)} \partial_{\alpha_{k+1}} \ldots \partial_{\alpha_{l}} O_{\alpha_{1} \ldots \alpha_{k}}(x)-\text { traces. }
$$

Here $\alpha^{\mathrm{S}}$ denotes symmetrization with respect to $\alpha_{1}, \ldots, \alpha_{l}$. As in the case of the collinear conformal representation, which is obtained by contraction with the light-cone vector $\tilde{n}$, the operator $O_{\alpha_{1} \ldots \alpha_{k}}(0)$ is the lowest member of the corresponding conformal tower. For dimensional reasons, the product of the currents $A$ and $B$ are expanded on the light cone as 


$$
\begin{aligned}
A(x) B(0)= & \sum_{k=0}^{\infty}\left(\frac{1}{x^{2}}\right)^{\left(l_{A}+l_{B}-l_{k}+k\right) / 2} \\
& \times \sum_{l=k}^{\infty} \widetilde{C}_{k l}(-i)^{(l+1)} x^{\alpha_{1}} \ldots x^{\alpha_{l}} \\
& \times O_{\alpha_{1} \ldots \alpha_{l}, k}(0)
\end{aligned}
$$

where $\widetilde{C}_{k l}$ are the Wilson coefficients. Furthermore, $l_{A}$ and $l_{B}$ denote the scale dimensions of the currents $A$ and $B$, respectively.

One way to construct the conformally covariant OPE is to act with $K_{\lambda}$ on both sides of the OPE (2.13) (see [10]) and compare the two results for the leading twist contributions. Taking into account the action of $K_{\lambda}$ on the currents

$$
\left[A(x) B(0), K_{\lambda}\right]=i\left(2 x_{\lambda}\left(l_{A}+x \partial\right)-x^{2} \partial_{\lambda}\right) A(x) B(0)
$$

and on the composite operators (2.12),

$$
\begin{gathered}
\left.\underset{\{\alpha, \lambda\}}{\mathrm{S}\left[O_{\alpha_{1}} \ldots \alpha_{l}, k\right.}(0), K_{\lambda}\right]=2(k-l)\left(l_{k}+l-1\right) \\
\quad \times \underset{\{\alpha, \lambda\}}{\mathrm{S}} O_{\alpha_{1} \ldots \alpha_{l-1}, k}(0) g_{\lambda \alpha_{l}}
\end{gathered}
$$

results, after comparison of the obtained expressions, into a recurrence relation for the Wilson coefficients:

$$
\begin{aligned}
\widetilde{C}_{k l+1}= & \frac{\left(l_{A}-l_{B}+l_{k}-k\right) / 2+l}{(l-k+1)\left(l_{k}+l\right)} \widetilde{C}_{k l} \\
& \Rightarrow \widetilde{C}_{k l}=\frac{\left(\left[l_{A}-l_{B}+l_{k}+k\right] / 2\right)_{l-k}}{(l-k) !\left(l_{k}+k\right)_{l-k}} \widetilde{C}_{k k} .
\end{aligned}
$$

Inserting this solution in the OPE (2.13) allows the summation with respect to $l$ and provides the conformally covariant OPE that is written here in the following representation:

$$
\begin{aligned}
A(x) B(0)= & \sum_{k=0}^{\infty} \widetilde{C}_{k}\left(\frac{1}{x^{2}}\right)^{\left(l_{A}+l_{B}-l_{k}+k\right) / 2} \\
& \times(-i)^{(k+1)} x^{\alpha_{1}} \ldots x^{\alpha_{k}} \\
& \times \int_{0}^{1} d u u^{\left(l_{A}-l_{B}+k+l_{k}\right) / 2-1} \\
& \times(1-u)^{\left(l_{B}-l_{A}+k+l_{k}\right) / 2-1} O_{\alpha_{1} \ldots \alpha_{k}}(u x),
\end{aligned}
$$

where $\widetilde{C}_{k}=\widetilde{C}_{k k}$.

Let us recall the assumptions used to derive this conformally covariant OPE (2.17). Besides the completeness of the operator basis, it was essential that $K_{\lambda}$ annihilates the currents $A, B$ and the conformal operators at the point $x_{\alpha}=0$ :

$$
\begin{aligned}
{\left[A(x), K_{\lambda}\right]_{\mid x=0}=\left[B(x), K_{\lambda}\right]_{\mid x=0} } & =0, \\
\left.\underset{\{\alpha, \lambda\}}{\mathrm{S}\left[O_{\alpha_{1}} \ldots \alpha_{k}\right.}(x), K_{\lambda}\right]_{\mid x=0} & =0 .
\end{aligned}
$$

The transformation laws under the infinitesimal conformal transformation (2.15), analogous to those in Eq. (2.10), result in a special arrangement of the operators. This causes the predictive power of the conformally covariant OPE, namely that the corresponding Wilson coefficients are already fixed and only the coefficients $\widetilde{C}_{k}$ are unknown and have to be computed explicitly, which can be done by forming forward matrix elements. In this case the $u$-dependence of the operators can be dropped and the conformal OPE is reduced to the common OPE for the forward case that is familiar from deep inelastic scattering (DIS).

\section{CONFORMALLY COVARIANT RENORMALIZATION SCHEME}

Generally, the conformal invariance of classical field theories is broken at the quantum level owing to the renormalization of the fields and the coupling constant. ${ }^{1}$ However, the symmetry breaking by the renormalization of the fields can be absorbed into the redefinition of the conformal representation, i.e. the scale dimension given originally by the canonical dimension is shifted by the anomalous dimension of the corresponding field. The renormalization of the coupling constant cannot be implemented in the original irreducible representation. However, in a scalar theory, Zaikov explored the possibility to extend the conformal representation of Green functions to a non-decomposable irreducible representation that includes the $\beta$-function [35]. In the following, it is simply assumed that there exists a non-trivial fixed point such that the $\beta$-function vanishes; formally, we speak from the conformal limit and set $\beta$ to zero.

The conformal properties of composite operators will also be spoiled by the renormalization. To study this symmetry breaking we employ the CWI [36], which was derived, for gauge field theories, in the canonical quantization [37] and in the path integral formulation [38]. Using the latter approach the CWI needed for conformal composite operators was written down in the dimensional regularization $[21,22]$. To have a convenient form for the Ward identities, we introduce a few shorthand notations. The symbol $[O]$ means renormalization of the operator $O$ in the $\overline{\mathrm{MS}}$ scheme. For simplicity we assume that the composite operators are closed under renormalization. Because of Poincaré invariance the $Z$-matrix is triangular (a detailed discussion on this point can be found, for instance, in [20]):

\footnotetext{
${ }^{1}$ In gauge field theories, the conformal invariance is also broken by the renormalization of the gauge-fixing parameter as well as explicitly by the gauge-fixing and ghost terms in the action. In the Abelian theory, this breaking can be formally written as a BecchiRouet-Stora-Tyutin (BRST) transformation, so that it does not appear in the physical sector of the theory. In the following we assume that this breaking is also absent in the physical sector of QCD.
} 


$$
\left[O_{k l}\right]=\sum_{k^{\prime}=0}^{k} Z_{k k^{\prime}} O_{k^{\prime} l}
$$

Furthermore, $X=\phi\left(x_{1}\right) \ldots \phi\left(x_{n}\right)$ is a monomial of elementary fields and $\langle A\rangle$ denotes the vacuum expectation value of the time-ordered product $\mathrm{T} A \exp i[S]$. Then the conformal Ward identities for the renormalized composite operators $\left[O_{k l}\right]\left(\nu_{1}, \nu_{2}\right)$ finally read $^{2}$

$$
\begin{aligned}
i \mathcal{D}\left\langle\left[O_{k l}\right] X\right\rangle= & \sum_{k^{\prime}=0}^{k}\left[\left(l+d_{1}^{\mathrm{can}}+d_{2}^{\mathrm{can}}\right) \delta_{k k^{\prime}}+\gamma_{k k^{\prime}}\right] \\
& \times\left\langle\left[O_{k^{\prime} l}\right] X\right\rangle+\frac{\beta}{g}\left\langle\left[O_{k l} \Delta^{\beta}\right] X\right\rangle+\cdots, \\
i \mathcal{K}_{-}\left\langle\left[O_{k l}\right] X\right\rangle= & -i \sum_{k^{\prime}=0}^{k}\left[2(k-l)\left(k+l+\nu_{1}+\nu_{2}\right) \delta_{k k^{\prime}}\right. \\
& \left.+\gamma_{k k^{\prime}}^{c}(l)\right]\left\langle\left[O_{k^{\prime} l-1}\right] X\right\rangle+\frac{\beta}{g}\left\langle\left[O_{k l} \Delta_{-}^{\beta}\right] X\right\rangle \\
& +\cdots .
\end{aligned}
$$

Here $\mathcal{D}$ and $\mathcal{K}$ are differential operators, which act on each field in the monomial $X$ as in Eq. (2.5), e.g.,

$$
\begin{aligned}
i \mathcal{D} & \left\langle\left[O_{k l}\right] \phi_{1}(x) \phi_{2}(y)\right\rangle \\
& =-\left(d_{1}+d_{2}+x \partial_{x}+y \partial_{y}\right)\left\langle\left[O_{k l}\right] \phi_{1}(x) \phi_{2}(y)\right\rangle,
\end{aligned}
$$

where the scale dimensions are shifted by the anomalous dimensions of the field, $d_{i}=d_{i}^{\text {can }}+\gamma_{i}$. The operators $\Delta^{\beta}$ and $\Delta_{-}^{\beta}=\tilde{n}^{\star} \mu \Delta_{\mu}^{\beta}$ arise from the conformal symmetry breaking in the action due to the renormalization of the coupling con- stant. Actually, they are given by the trace anomaly of the energy-momentum tensor [39-42] and will be written here in terms of the renormalized Lagrangian $\mathcal{L}(x)[37,38]$ :

$$
\left[\Delta^{\beta}\right]=i \int d^{n} x g \frac{\partial}{\partial g} \mathcal{L}(x), \quad\left[\Delta_{\lambda}^{\beta}\right]=i \int d^{n} x 2 x_{\lambda} g \frac{\partial}{\partial g} \mathcal{L}(x) .
$$

The ellipses in the CWI denote Green functions with operator insertions caused by the gauge-fixing and ghost terms. Such contributions should be absent in physical matrix elements. The expression $\gamma_{k k^{\prime}}$ is the anomalous-dimension matrix of the operators and $\gamma_{k k^{\prime}}^{c}(l)$ denotes the specialconformal anomaly matrix, which breaks the covariance of the operators under infinitesimal special conformal transformations. Such transformations break the Poincaré invariance ( $K_{\alpha}$ does not commute with $M_{\beta \gamma}$ and $P_{\beta}$ ) and therefore the spin $l$ dependence appears.

\section{A. Leading order analysis}

It is well known that the anomalous-dimension matrix of conformal two-particle operators is diagonal to LO: $\gamma_{k k^{\prime}}^{(0)}$ $=\gamma_{k}^{(0)} \delta_{k k^{\prime}}$. However, in a general renormalization scheme the irreducible conformal representation is already broken by an off-diagonal special-conformal anomaly matrix. Using the dimensional regularization and the $\overline{\mathrm{MS}}$ prescription the results for the $\phi_{(6)}^{3}$ theory read, in matrix notation [21]:

$$
\begin{aligned}
\hat{\gamma}^{c(0)}(l) & =-\hat{b}(l) \hat{\gamma}^{(0)}, \quad \hat{b}(l)=\left\{b_{k k^{\prime}}(l)\right\}, \\
\hat{\gamma}^{(0)} & =\left\{\gamma_{k}^{(0)} \delta_{k k^{\prime}}\right\},
\end{aligned}
$$

where

$$
b_{k k^{\prime}}(l)=\left\{\begin{array}{l}
2\left(l+k^{\prime}+3\right) \delta_{k k^{\prime}}-2\left(2 k^{\prime}+3\right) \quad \text { if } k-k^{\prime} \geq 0 \text { and even } \\
0 \text { otherwise. }
\end{array}\right.
$$

Regularization of the "gluon" propagator via Pauli-Villars provides a different breaking of the special conformal transformation:

$$
\hat{\gamma}^{c(0)}(l)=-\hat{\gamma}^{(0)} \hat{b}(l)
$$

However, this breaking can easily be absorbed in a redefini-

\footnotetext{
${ }^{2}$ These CWI do not rely on any assumptions about the conformal symmetry breaking and they differ from the ones used in a previous study in Ref. [14] by the triangularity of the anomalous matrices.
}

tion of the local operator by changing the $\operatorname{index}^{3} \nu \rightarrow \nu$ $-\left(\alpha_{s} / 4 \pi\right) \gamma_{k}^{(0)}$ and it coincides with the prediction of conformal symmetry [20].

For the conformal flavor non-singlet quark operators in QCD the symmetry breaking is even more complicated [22] due to the covariant derivatives:

$$
\hat{\gamma}^{c(0)}(l)=-\hat{b}(l) \hat{\gamma}^{(0)}+\hat{w}
$$

\footnotetext{
${ }^{3}$ Here $\alpha_{s}$ denotes of course an appropriate definition of the coupling constant in the scalar theory. Note that only at one-loop order the conformal symmetry prediction for the eigenfunctions of the evolution kernel coincides with the shift of the index $\nu$ for the operator.
} 
where the non-vanishing elements of $w_{k k^{\prime}}$ (for $k-k^{\prime}>0$ and $k-k^{\prime}$ even) are

$$
\begin{aligned}
w_{k k^{\prime}}= & -4 C_{F}\left(2 k^{\prime}+3\right)\left(k-k^{\prime}\right)\left(k+k^{\prime}+3\right) \\
& \times\left[\frac{A_{k k^{\prime}}-\psi(k+1)+\psi(0)}{\left(k^{\prime}+1\right)\left(k^{\prime}+2\right)}\right. \\
& \left.+\frac{2 A_{k k^{\prime}}}{\left(k-k^{\prime}\right)\left(k+k^{\prime}+3\right)}\right], \\
A_{k k^{\prime}}= & \psi\left(\frac{k+k^{\prime}+4}{2}\right)-\psi\left(\frac{k-k^{\prime}}{2}\right) \\
& +2 \psi\left(k-k^{\prime}\right)-\psi(k+2)-\psi(1),
\end{aligned}
$$

with $\psi(z)=(d / d z) \ln \Gamma(z)$. It turns out that the appearance of the matrix $\hat{w}$ explains the difference between the conformal symmetry prediction for the eigenfunctions of the pion evolution kernel and the explicit NLO calculation.

Normalization conditions, which are given implicitly in the $\overline{\mathrm{MS}}$ scheme, are a matter of convenience, and changing them does not affect physical quantities. Thus we can look for a scheme in which conformal covariance is restored. Such a scheme can be obtained by a finite renormalization; at LO we define the renormalized conformally covariant operators as

$$
\begin{aligned}
O_{k l}^{\mathrm{co}}= & {\left[O_{k l}\right]-\frac{\alpha_{s}}{2 \pi} \sum_{k^{\prime}=0}^{k-2} \frac{\gamma_{k k^{\prime}}^{c(0)}(l)}{2\left(k^{\prime}-k\right)\left(k^{\prime}+k+\nu_{1}+\nu_{2}\right)} } \\
& \times\left[O_{k^{\prime} l}\right]+\ldots, \\
= & O_{k l}+\frac{\alpha_{s}}{2 \pi} \frac{\gamma_{k}^{(0)}}{2 \epsilon} O_{k l}-\frac{\alpha_{s}}{2 \pi} \sum_{k^{\prime}=0}^{k-2} \\
& \times \frac{\gamma_{k k^{\prime}}^{c(0)}(l)}{2\left(k^{\prime}-k\right)\left(k^{\prime}+k+\nu_{1}+\nu_{2}\right)} O_{k^{\prime} l}+\ldots,
\end{aligned}
$$

where $1 / \epsilon$ is the usual $\epsilon$-pole in dimensional regularization, which satisfy the CWI:

$$
\begin{aligned}
i \mathcal{D}\left\langle O_{k l}^{\mathrm{co}} X\right\rangle= & {\left[l+d_{1}^{\mathrm{can}}+d_{2}^{\mathrm{can}}+\frac{\alpha_{s}}{2 \pi} \gamma_{k}^{(0)}\right]\left\langle O_{k l}^{\mathrm{co}} X\right\rangle+\cdots, } \\
i \mathcal{K}_{-}\left\langle O_{k l}^{\mathrm{co}} X\right\rangle= & -i\left[2(k-l)\left(k+l+\nu_{1}+\nu_{2}\right)\right. \\
& \left.+\frac{\alpha_{s}}{2 \pi} \gamma_{k k}^{c(0)}(l)\right]\left\langle O_{k l-1}^{\mathrm{co}} X\right\rangle+\cdots
\end{aligned}
$$

For the scalar theory as well as for QCD we find from Eqs. (3.5)-(3.8) that $\gamma_{k k}^{c(0)}(l)=2(k-l) \gamma_{k}^{(0)}$. Thus, in both equations of the CWI (3.11) the conformal symmetry breaking by the anomalous dimension is absorbed into the shift of the canonical dimension of the operators $l+d_{1}^{\text {can }}+d_{2}^{\text {can }} \rightarrow l$ $+d_{1}^{\text {can }}+d_{2}^{\text {can }}+\gamma_{k}$. In this way the irreducible conformal representation is restored for the renormalized operator at LO.
So as conformal covariance at tree level is sufficient for a diagonal anomalous-dimension matrix at LO, the one-loop renormalized conformal operators do not mix under renormalization in NLO, of course, up to a term proportional to $\beta_{0}$.

\section{B. Restoration of conformal covariance}

Before we use conformal constraints to extend the analysis to the full conformal theory, let us show that the property $\gamma_{k k}^{c(0)}(k)=0$ holds true generally. To make the discussion transparent, let us first consider the scalar theory in which $\hat{\gamma}^{c}(l)$ is defined in the $\overline{\mathrm{MS}}$ scheme as [21]

$$
\hat{\gamma}^{c}(l)=-2 \gamma_{\varphi} \hat{b}(l)+2\left[\hat{Z}^{[1]}, \hat{b}(l)\right]+\hat{Z}^{\star[1]}(l),
$$

where the counterterm $\hat{Z}^{\star}=\hat{Z}^{\star[1]} / \epsilon+\hat{Z}^{\star[2]} / \epsilon^{2}+\cdots$ has to be computed from the renormalization of the operator product

$$
\left[O_{k l}\right]\left[\Delta_{-}^{\beta}\right]=\left[O_{k l} \Delta_{-}^{\beta}\right]+i \sum_{k^{\prime}=0}^{k} Z_{k k^{\prime}}^{\star}(l)\left[O_{k^{\prime} l}\right]
$$

From the properties of $\hat{b}(l)$ and $\hat{Z}$ it follows that $\gamma_{k k}^{c}(k)$ $=Z_{k k}^{\star[1]}(k)$. The latter is determined by the UV-divergent part of $\int d^{n} x\left(\tilde{n}^{\star} x\right) \varphi^{3}(x) O_{k k}$. Since $O_{k k}$ is a polynomial of order $k$ in the derivatives and the UV divergence is concentrated in $x^{\alpha}=0$, it is clear that $\tilde{n}^{\star} x$ annihilates one derivative, so that a polynomial of order $k-1$ remains. Thus, no counterterm $O_{k k}$ is needed and therefore $\gamma_{k k}^{c}(k)=\hat{Z}_{k k}^{\star[1]}(k)=0$. In gauge field theories the definition of $\hat{Z}^{\star}$ is modified by a term containing the functional derivative with respect to the gauge field [22]: $\int d^{n} x\left(\tilde{n}^{*} x\right) A_{\mu}\left(\delta / \delta A_{\mu}\right)\left[O_{k k}\right]$. Obviously, we can use the same arguments as above, and this term also does not induce a contribution to $\gamma_{k k}^{c}(k)$.

The proof that conformal covariance can be restored in the conformal limit will be achieved in the following manner. First we notice that the anomalous-dimension matrix will be diagonalized by a finite renormalization group transformation,

$$
i \mathcal{D}\left\langle O_{k l}^{\mathrm{co}} X\right\rangle=\left(l+d_{1}^{\mathrm{can}}+d_{2}^{\mathrm{can}}+\gamma_{k}\right)\left\langle O_{k l}^{\mathrm{co}} X\right\rangle+\cdots
$$

Then we show, with the help of conformal constraints, that this implies

$$
\begin{aligned}
i \mathcal{K}_{-}\left\langle O_{k l}^{\mathrm{co}} X\right\rangle= & -2 i(k-l)\left[k+l+\nu_{1}+\nu_{2}+\gamma_{k}\right] \\
& \times\left\langle O_{k l-1}^{\mathrm{co}} X\right\rangle+\cdots .
\end{aligned}
$$

To calculate the right-hand side (RHS) of Eq. (3.15) we solve the conformal constraints in the following subsection. In a second subsection we consider the flavor-singlet channel in QCD, where an additional mixing problem between quark and gluon operators appears. Here, we only take into account the algebraic properties of the constraints and the triangularity of the matrices to show that Eq. (3.14) implies Eq. (3.15). 


\section{Solution of the conformal constraints}

A constraint for the anomalous-dimension matrix, which allows the off-diagonal part to be computed in terms of the special-conformal anomaly matrix, is implied by the commutator relation

$$
\left[\mathcal{D}, \mathcal{K}_{-}\right]=i \mathcal{K}_{-}
$$

Applying this identity to the Green functions and using the CWI provides immediately a commutator relation for the anomalous-dimension matrix and the special-conformal anomaly matrix. For completeness, we give the exact result, which includes the full $\beta$-dependence $[21,22]$ in the dimensional regularization for the $\overline{\mathrm{MS}}$ prescription: ${ }^{4}$

$$
\begin{gathered}
{\left[\hat{a}(l)+\hat{\gamma}^{c}(l)+2 \frac{\beta}{g} \hat{b}(l), \hat{\gamma}\right]=0,} \\
a_{k k^{\prime}}(l)=2(k-l)\left(k+l+\nu_{1}+\nu_{2}\right) \delta_{k k^{\prime}} .
\end{gathered}
$$

Since the matrix $\hat{a}$ is diagonal, a recurrence relation follows for the off-diagonal part $\hat{\gamma}^{N D}$ of the anomalous-dimension matrix ( $\beta$ is now consequently set to zero):

$$
\gamma_{k k^{\prime}}^{N D}=-\left\{\mathcal{G} \hat{\gamma}^{D}\right\}_{k k^{\prime}}-\left\{\mathcal{G} \hat{\gamma}^{N D}\right\}_{k k^{\prime}}
$$

Here $\hat{\gamma}^{D}=\left\{\gamma_{k} \delta_{k k^{\prime}}\right\}$ denotes the diagonal part of the anomalous-dimension matrix and the operator $\mathcal{G}$ is defined by

$$
\mathcal{G} \hat{A}:= \begin{cases}\frac{\left[\hat{\gamma}^{c}(l), \hat{A}\right]_{k k^{\prime}}}{2\left(k-k^{\prime}\right)\left(k+k^{\prime}+\nu_{1}+\nu_{2}\right)} & \text { if } k-k^{\prime}>0 \\ 0 \quad \text { otherwise. } & \end{cases}
$$

The solution of Eq. (3.18) can be formally written as

$$
\hat{\gamma}^{N D}=-\frac{\mathcal{G}}{\hat{1}+\mathcal{G}} \hat{\gamma}^{D}=-\mathcal{G} \hat{\gamma}^{D}+\mathcal{G}^{2} \hat{\gamma}^{D}-\cdots
$$

The composite operators, which do not mix under renormalization, are obtained by a finite renormalization

$$
O_{k l}^{\mathrm{co}}=\sum_{k^{\prime}=0}^{k} B_{k k^{\prime}}^{-1}\left[O_{k^{\prime} l}\right]
$$

The matrix $\hat{B}=\left\{B_{k k^{\prime}}\right\}$ can be calculated from

\footnotetext{
${ }^{4}$ The derivation is tricky and all details for the scalar theory in dimensional regularization are given in [21]. The calculation for the Abelian gauge field theory is analogous and as expected leads to no explicit gauge dependence in the commutator relation. Since $\hat{\gamma}$ is a physical quantity we can assume that, at least for $\beta=0$, this constraint holds also true in QCD.
}

$$
\hat{\gamma}^{D}=\hat{B}^{-1} \hat{\gamma} \hat{B} \Rightarrow\left[\hat{B}, \hat{\gamma}^{D}\right]=\hat{\gamma}^{\mathrm{ND}} \hat{B}
$$

where the diagonal matrix $\hat{\gamma}^{D}$ consists of the eigenvalues of the triangular anomalous-dimension matrix. The solution of this equation is

$$
\hat{B}=\frac{\hat{1}}{\hat{1}-\mathcal{L} \hat{\gamma}^{\mathrm{ND}}}=\hat{1}+\mathcal{L} \hat{\gamma}^{\mathrm{ND}}+\mathcal{L}\left(\hat{\gamma}^{\mathrm{ND}} \mathcal{L} \hat{\gamma}^{\mathrm{ND}}\right)+\cdots
$$

where the operator $\mathcal{L}$ is defined by

$$
\mathcal{L} \hat{A}:=\left\{\begin{array}{l}
-\frac{A_{k k^{\prime}}}{\gamma_{k}-\gamma_{k^{\prime}}} \text { if } k-k^{\prime}>0 \\
0 \quad \text { otherwise. }
\end{array}\right.
$$

The off-diagonal matrix $\hat{\gamma}^{\mathrm{ND}}$ is given in terms of $\hat{\gamma}^{\mathrm{c}}$, implying that the transformation matrix $\hat{B}$ can also be expressed by the special-conformal anomaly matrix. From Eqs. (3.20) and (3.23) one finds, after some algebra, that the diagonal anomalous-dimension matrix cancels out (see Appendix A):

$$
\hat{B}=\frac{\hat{1}}{\hat{1}+\mathcal{J} \hat{\gamma}^{c}}=\hat{1}-\mathcal{J} \hat{\gamma}^{c}+\mathcal{J}\left(\hat{\gamma}^{c} \mathcal{J} \hat{\gamma}^{c}\right)-\cdots,
$$

where the operator $\mathcal{J}$ is defined by

$$
\mathcal{J A}:=\left\{\begin{array}{l}
\frac{A_{k k^{\prime}}}{2\left(k-k^{\prime}\right)\left(k+k^{\prime}+\nu_{1}+\nu_{2}\right)} \text { if } k-k^{\prime}>0, \\
0 \quad \text { otherwise. }
\end{array}\right.
$$

Note that in the forward case all operators $O_{k l}$ with $l>k$ vanish. Thus, the renormalization group transformation (3.21) does not affect the minimal subtraction prescription in the forward case: $O_{k k}^{\text {co }}=\left[O_{k k}\right]$.

Now we are almost able to prove that the operators $O_{k l}^{\text {co }}$ are conformally covariant. For this purpose we need the spin dependence of the special-conformal anomaly matrix, which is constrained by the commutator relation:

$$
\left[\mathcal{K}_{-}, \mathcal{P}_{+}\right]=-2 i\left(\mathcal{D}+\mathcal{M}_{-+}\right) \text {. }
$$

Applying this relation to the Green functions and using the CWI (3.2) provides

$$
\hat{\gamma}^{c}(l+1)-\hat{\gamma}^{c}(l)=-2 \hat{\gamma}
$$

The solution of this recurrence relation gives the spin dependence of the special-conformal anomaly matrix

$$
\gamma_{k k^{\prime}}^{c}(l)=\gamma_{k k^{\prime}}^{c}(k)+2(k-l) \gamma_{k k^{\prime}},
$$

and for $k=k^{\prime}: \quad \gamma_{k k}^{c}(l)=2(k-l) \gamma_{k}$,

where the last equation follows from the above shown property $\gamma_{k k}^{c}(k)=0$. The special conformal anomaly of the operators $O_{k l}^{\text {co }}$ are given by $\hat{B}^{-1}\left[\hat{a}(l)+\hat{\gamma}^{c}(l)\right] \hat{B}$ and using the cor- 
responding definitions and the property (3.29), a straightforward calculation given in the Appendix A provides

$$
\begin{aligned}
\left\{\hat{B}^{-1}\left[\hat{a}(l)+\hat{\gamma}^{c}(l)\right] \hat{B}\right\}_{k k^{\prime}}= & 2(k-l)\left(k+l+\nu_{1}+\nu_{2}\right. \\
& \left.+\gamma_{k}\right) \delta_{k k^{\prime}},
\end{aligned}
$$

which is equivalent to Eq. (3.15).

\section{Additional mixing problem in the QCD singlet channel}

The leading twist operators appearing in the singlet channel can be written in the following conformally covariant manner, where for even parity we have:

$$
\begin{aligned}
& { }^{q} O_{k l}=\partial_{+}^{l} \bar{\psi} \tilde{n}_{\alpha} \gamma^{\alpha} C_{k}^{3 / 2}\left(\frac{\tilde{n} D_{-}}{\tilde{n} \partial_{+}}\right) \psi, \quad l \geqslant k \geqslant 0, \quad(3.31) \\
& { }^{g} O_{k l}=\partial_{+}^{l-1} \tilde{n}^{\alpha} F_{\alpha \beta} C_{k-1}^{5 / 2}\left(\frac{\tilde{n} D_{-}}{\tilde{n} \partial_{+}}\right) F^{\beta \gamma} \tilde{n}_{\gamma}, \quad l \geqslant k \geqslant 1,
\end{aligned}
$$

while for odd parity:

$$
\begin{aligned}
& { }^{q} R_{k l}=\partial_{+}^{l} \bar{\psi} \tilde{n}_{\alpha} \gamma^{\alpha} \gamma^{5} C_{k}^{3 / 2}\left(\frac{\tilde{n} D_{-}}{\tilde{n} \partial_{+}}\right) \psi, \quad l \geqslant k \geqslant 0, \\
& { }^{g} R_{k l}=\partial_{+}^{l-1} \tilde{n}^{\alpha} \widetilde{F}_{\alpha \beta} C_{k-1}^{5 / 2}\left(\frac{\tilde{n} D_{-}}{\tilde{n} \partial_{+}}\right) F^{\beta \gamma} \tilde{n}_{\gamma}, \quad l \geqslant k \geqslant 1 .
\end{aligned}
$$

Here flavor and color indices are suppressed for simplicity, $D_{-}^{\nu}=\vec{D}^{\nu}-\overleftarrow{D}^{\nu}$ are the covariant derivatives, $F_{\alpha \beta}$ and $\widetilde{F}_{\alpha \beta}$ $=\epsilon_{\alpha \beta \gamma \delta} F^{\gamma \delta} / 2$ are the field strength and the dual-field strength tensor, respectively. Since the dimension of the field strength tensor is 2 and its spin is 1 the index of the Gegenbauer polynomials for the gluon operators is $\nu=5 / 2$. All these operators have spin $l+1$ and canonical dimension $l+3$, as well as the same behavior under special conformal transformations at tree level.

The following discussion is valid for operators of even and odd parity. The quark and gluon operators will mix, and the anomalous-dimension matrix of the operators can therefore be written in the following compact notation:

$$
\hat{\gamma}=\left(\begin{array}{c}
q q \\
{ }^{q g} \hat{\gamma} \\
{ }^{g q} \hat{\gamma}^{g g} \hat{\gamma}
\end{array}\right),
$$

where the entries ${ }^{i j} \hat{\gamma}$ for $i, j=\{q, g\}$ are triangular matrices. At LO these entries are diagonal and the remaining mixing problem has to be solved by explicit diagonalization of the $2 \times 2$ matrix.

With the previous assumption about the unphysical part in the CWI, the generalization of the conformal constraint (3.17) for $\beta=0$ is a purely algebraic task:

$$
\left[\left(\begin{array}{cc}
\hat{a}(l) & 0 \\
0 & \hat{a}(l)
\end{array}\right)+\left(\begin{array}{c}
{ }^{q q} \hat{\gamma}^{c}(l)^{q g} \hat{\gamma}^{c}(l) \\
{ }^{g q} \hat{\gamma}^{c}(l)^{g g} \hat{\gamma}^{c}(l)
\end{array}\right),\left(\begin{array}{c}
{ }^{q q} \hat{\gamma}^{q g} \hat{\gamma} \\
{ }^{g q} \hat{\gamma}^{g g} \hat{\gamma}
\end{array}\right)\right]=0,
$$

where $a_{k k^{\prime}}(l)=2(k-l)(k+l+3) \delta_{k k^{\prime}}$. Now we introduce the matrix

$$
\hat{B}=\left(\begin{array}{c}
{ }^{q q} \hat{B}^{q g} \hat{B} \\
{ }^{g q} \hat{B}^{g g} \hat{B}
\end{array}\right),
$$

implying that the anomalous-dimension matrices of the operators

$$
\begin{aligned}
& { }^{q} O_{k l}^{\mathrm{co}}=\sum_{k^{\prime}=0}^{k}\left({ }^{q q} B_{k k^{\prime}}^{-1}\left[{ }^{q} O_{k^{\prime} l}\right]+{ }^{q g} B_{k k^{\prime}}^{-1}\left[{ }^{g} O_{k^{\prime} l}\right]\right), \\
& { }^{g} O_{k l}^{\mathrm{co}}=\sum_{k^{\prime}=0}^{k}\left({ }^{g q} B_{k k^{\prime}}^{-1}\left[{ }^{q} O_{k^{\prime} l}\right]+{ }^{g g} B_{k k^{\prime}}^{-1}\left[{ }^{g} O_{k^{\prime} l}\right]\right)
\end{aligned}
$$

consist only of diagonal entries

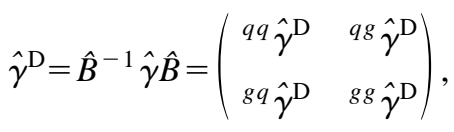

where ${ }^{i j} \gamma_{k k^{\prime}}^{\mathrm{D}}={ }^{i j} \gamma_{k} \delta_{k k^{\prime}}$. Applying the transformation (3.39) to the conformal constraint (3.36) tells us that

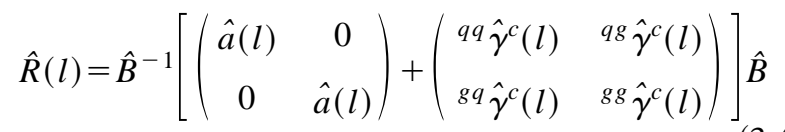

possesses only diagonal entries. Taking into account the property $\hat{a}(l+1)-\hat{a}(l)=-2(l+3) \hat{1}$ and the analogous equation to Eq. (3.28) it follows that

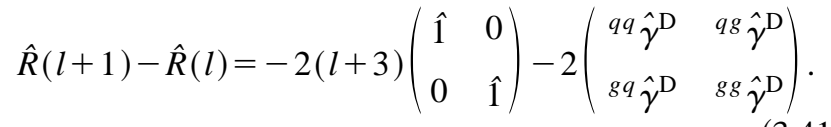

The solution of this recurrence relation together with the property $R_{k k}(k)=0$ for $i, j=\{q, g\}$ gives the following term in the RHS of the special conformal Ward identity:

$$
R_{k k^{\prime}}(l)=2(k-l)\left(\begin{array}{cc}
k+l+3+{ }^{q q} \gamma_{k} & { }^{q g} \gamma_{k} \\
{ }^{g q} \gamma_{k} & k+l+3+{ }^{g g} \gamma_{k}
\end{array}\right) \delta_{k k^{\prime}},
$$

which shows that the lowest member of each tower will be annihilated by the action of $K_{-}$in the conformal limit of the theory.

To restore the conformal covariance completely, the remaining mixing problem that is well known from the forward case has to be solved by introducing the eigenvectors:

$$
{ }^{+} O_{k l}^{\mathrm{co}}={ }^{q} O_{k l}^{\mathrm{co}}+C_{k}^{+g} O_{k l}^{\mathrm{co}}, \quad{ }^{-} O_{k l}^{\mathrm{co}}={ }^{g} O_{k l}^{\mathrm{co}}+C_{k}^{-q} O_{k l}^{\mathrm{co}} .
$$


These operators have completely diagonal anomalous dimension and special conformal anomaly matrices:

$$
\hat{\gamma}=\left(\begin{array}{cc}
+\hat{\gamma}^{\mathrm{D}} & 0 \\
0 & -\hat{\gamma}^{\mathrm{D}}
\end{array}\right), \quad \hat{\gamma}^{\mathrm{c}}=2(k-l)\left(\begin{array}{cc}
+\hat{\gamma}^{\mathrm{D}} & 0 \\
0 & -\hat{\gamma}^{\mathrm{D}}
\end{array}\right) .
$$

\section{Conformally covariant OPE in the interacting theory}

In the previous subsection it has been proved that there exists, in the hypothetical conformal limit of the theory, a renormalization scheme in which the conformal covariance of the renormalized operators is ensured. This scheme is related to any other one by a finite renormalization of the composite operators, where, however, in the forward case the normalization of these operators remains unchanged. In the OPE the renormalized operators are normalized at the factorization scale. These normalization conditions are arbitrary and the physical quantities, which are defined in terms of the product of two currents, are independent of these conditions. Now we choose such normalization conditions that ensure the covariance of the renormalized conformal operators.

Because of the covariance, the operators in different towers do not mix under dilatations, and the dilatation invariance requires the form of the OPE given in Eq. (2.13), where the scaling dimension of the renormalized currents and of the renormalized conformal operators is now $l_{i}=l_{i}^{\text {can }}+\gamma_{i}$ for $i$ $=\{A, B\}$ and $l_{k}=d_{1}^{\text {can }}+d_{2}^{\text {can }}+k+\gamma_{k}$, respectively. Furthermore, the renormalized conformal operators transform under infinitesimal special conformal transformations formally as in Eq. (2.15), so that the lowest member of each conformal tower will be annihilated by $K_{\lambda}$. Hence, we can apply the same algebraic steps as previously; the result will be the same formal expression as Eq. (2.17) also for the interacting theory, however, with shifted scale dimensions:

$$
\begin{aligned}
A(x) B(0)= & \sum_{k=0}^{\infty} \widetilde{C}_{k}(\mu)\left(\frac{1}{x^{2}}\right)^{\left(l_{A}+l_{B}-d_{1}^{\mathrm{can}}-d_{2}^{\mathrm{can}}-\gamma_{k}\right) / 2}(-i)^{(k+1)} x^{\alpha_{1}} \ldots x^{\alpha_{k}} \\
& \times \int_{0}^{1} d u u^{\left(l_{A}-l_{B}\right) / 2}(1-u)^{\left(l_{B}-l_{A}\right) / 2}[u(1-u)]^{\left(d_{1}^{\mathrm{can}}+d_{2}^{\mathrm{can}}+\gamma_{k}\right) / 2+k-1} O_{\alpha_{1}}^{\mathrm{co}} \ldots \alpha_{k}(u x)_{\mu} .
\end{aligned}
$$

In the conformal factorization scheme, the Wilson coefficients and the composite operators satisfy simple renormalization group equations:

$$
\begin{aligned}
\mu \frac{\partial}{\partial \mu} \widetilde{C}_{k}(\mu) & =\left(\gamma_{k}-\gamma_{A}-\gamma_{B}\right) \widetilde{C}_{k}(\mu), \\
\mu \frac{\partial}{\partial \mu} O_{\alpha_{1} \ldots \alpha_{k}}^{\mathrm{co}}(0)_{\mu} & =-\gamma_{k} O_{\alpha_{1} \ldots \alpha_{k}}^{\mathrm{co}}(0)_{\mu} .
\end{aligned}
$$

The conformally covariant OPE for the product of two electromagnetic currents in QCD should be constructed at leading twist-2 in an analogous way. However, to avoid technical complications due to the Lorentz structure and the gauge-invariant decomposition, we consider here only two independent contributions, namely the trace $J_{\mu}(x) J^{\mu}(0)$ and the antisymmetric twist-2 part $\left\{J_{\mu}(x) J_{\nu}(0)\right\}^{\text {asy }}$ proportional to the $\epsilon$-tensor. The neglected twist-3 contributions induce in certain cases nonpower-suppressed contributions as for the structure function $g_{2}$ in polarized DIS. In both cases considered, the Lorentz structure does not affect the derivation of the conformal OPE, and we can proceed in principle as in the scalar theory. Of course, the conformal OPE is separately valid for the flavor non-singlet and singlet channel. The appearing mixing problem in the singlet channel can be resolved easily, as discussed above, by introducing appropriate linear combinations of quark and gluon operators, which will be considered as independent. Hence, in the conformal limit, each Wilson coefficient that appears can be written in the same form as given in Eq. (3.45):

$$
\begin{aligned}
\left\{\begin{array}{c}
J_{\mu}(x) J^{\mu}(0) \\
\left\{J_{\mu}(x) J_{\nu}(0)\right\}^{\text {asy }}
\end{array}\right\}= & \sum_{k=0}^{\infty} \sum_{i}\left\{\begin{array}{cc}
x^{\alpha_{1}} & { }^{i} \widetilde{C}_{k}(\mu) \\
i \epsilon_{\mu \nu \lambda}{ }^{\alpha} x^{\lambda} & { }^{i} \widetilde{E}_{k}(\mu)
\end{array}\right\}\left(\frac{1}{x^{2}}\right)^{2-1 / 2 i} \gamma_{k}(-i)^{k+1} x^{\alpha_{2}} \ldots x^{\alpha_{k}+1} \\
& \times \int_{0}^{1} d u[u(1-u)]^{k+1+1 / 2 i} \gamma_{k}\left\{\begin{array}{c}
{ }^{i} O_{\alpha_{1} \ldots \alpha_{k+1}}^{\mathrm{co}}(u x) \\
{ }^{i} R_{\alpha_{1} \ldots \alpha_{k+1}}^{\mathrm{co}}(u x)
\end{array}\right\},
\end{aligned}
$$


where the index $i=\{\mathrm{NS},+,-\}$ denotes the quark operators in the non-singlet channel as well as the eigenvectors introduced in the singlet channel. Note that the anomalous dimensions ${ }^{i} \gamma_{k}$ are different for even and odd parity operators.

To compute the Wilson coefficients one would choose, for practical purposes, the simplest normalization conditions. Then the conformal covariance of the OPE is not manifest and the operators will mix under renormalization. However, putting together the solution of the more complicated renormalization group equation and the Wilson coefficients provides the same scheme-independent result as the conformally covariant OPE. This fact will be used below for a consistency check of the available non-forward and forward QCD calculations for the product of two electromagnetic currents in NLO.

\section{CONFORMAL PREDICTION FOR TWO-PHOTON PROCESSES IN THE LIGHT-CONE-DOMINATED REGION}

In the following we consider two-photon processes, where at least one of the photons is far off-shell, so that the momentum transfer is large, which means that the distance between the photons is light-like. Such processes are the deeply virtual Compton scattering (DVCS), which is widely discussed at present [24-26]:

$$
\gamma^{*}\left(q_{1}\right)+\mathrm{H}\left(P_{1}\right) \rightarrow \gamma^{*}\left(q_{2}\right)+\mathrm{H}\left(P_{2}\right),
$$

and the production of some hadronic final states by photonphoton collision, e.g. the crossed process to the DVCS:

$$
\gamma^{*}\left(q_{1}\right)+\gamma^{*}\left(q_{2}\right) \rightarrow \mathrm{H}\left(P_{1}\right)+\mathrm{H}\left(P_{2}\right) .
$$

We pay special attention to meson transition form factors $\gamma^{*}+\gamma^{*} \rightarrow \mathrm{M}$. Note that also the production of two jets in the light-cone-dominated region was already considered in the beginning of the 1980's by Chase [43,44]. Both processes (4.1) and (4.2) were previously studied in the framework of the non-local light-cone expansion by Geyer, Robaschik and collaborators at leading order $[45,23]$. Here we employ the conformally covariant OPE to predict the leading twist-2 contributions of these non-forward processes restricted to the conformal limit in terms of the off-diagonal expectation values of composite operators.

\section{A. General formalism}

The scattering amplitude for two-photon processes is given by the time-ordered product of two electromagnetic currents sandwiched between the corresponding hadronic states. To be more general, we define the scattering amplitude in the momentum space generically as

$$
T\left(P_{1}, P_{2}, q\right)=i \int d^{n} x e^{i q x}\left\langle P_{2}\left|T J\left(\frac{x}{2}\right) J\left(\frac{-x}{2}\right)\right| P_{1}\right\rangle,
$$

where $J$ denotes a current and $n$ is again the (integer) spacetime dimension. At large momentum transfer $q=\left(q_{1}\right.$ $\left.+q_{2}\right) / 2$ the process is dominated by the contributions from the light cone and we can define the following generalized Bjorken region $[45,23]$ :

$$
\nu=P q \rightarrow \infty, \quad \text { where } P=P_{1}+P_{2}, \quad Q^{2}=-q^{2} \rightarrow \infty,
$$

with the scaling variables

$$
\xi=\frac{1}{\omega}=\frac{-q^{2}}{P q}, \quad \eta=\frac{\Delta q}{P q}, \quad \text { where } \Delta=P_{2}-P_{1} .
$$

In the forward case $\xi$ is identical to the Bjorken variable $x_{B j}$, and $\eta$ vanishes. For non-forward Compton scattering in the Breit frame $\eta$ is approximately given by $\cos \phi=-(\vec{\Delta} \vec{q}|| \vec{\Delta} \| \vec{q} \mid)[45,23]$. Formally, $\eta$ interpolates between different processes, for instance the two-photon production of one hadron requires $\eta=1$.

It is straightforward to derive the conformal predictions for the two-photon processes in the generalized Bjorken limit by inserting the conformally covariant OPE into the scattering amplitude (4.3):

$$
\begin{aligned}
T\left(\omega, \eta, Q^{2}\right)= & \int d^{n} x e^{i\{q x-1 / 2 \Delta x\}} \sum_{k=0}^{\infty} \widetilde{C}_{k}(\mu)\left(\frac{1}{x^{2}}\right)^{\left(2 l_{J}-l_{k}+k\right) / 2} \\
& \times(-i)^{k} x^{\alpha_{1}} \ldots x^{\alpha_{k}} \int_{0}^{1} d u[u(1-u)]^{\left(l_{k}+k\right) / 2-1} \\
& \times\left\langle P_{2}\left|O_{\alpha_{1}}^{\mathrm{co}} \ldots \alpha_{k}(u x)\right| P_{1}\right\rangle .
\end{aligned}
$$

The expectation value $\left\langle P_{2}\left|O_{\alpha_{1} \ldots \alpha_{k}}^{\text {co }}(u x)\right| P_{1}\right\rangle$ is a symmetric and traceless tensor, which can be built from the vectors $P_{\alpha_{i}}$ and $\Delta_{\alpha_{i}}$. With respect to the Fourier transform, where we keep only the leading terms in $Q^{2}$, we already set $\Delta x$ $=\eta P x$ as well as $\Delta_{\alpha_{i}}=\eta P_{\alpha_{i}}$ :

$$
\begin{aligned}
\left\langle P_{2}\left|O_{\alpha_{1} \ldots \alpha_{k}}^{\mathrm{co}}(u x)\right| P_{1}\right\rangle_{\mu}= & P_{\alpha_{1}} \ldots P_{\alpha_{k}} e^{i u \eta(P x)} \\
& \times\left\langle P_{2}\left|O_{k}^{\mathrm{co}}(0)\right| P_{1}\right\rangle_{\mu}(\eta) .
\end{aligned}
$$

These reduced expectation values are polynomials of order $k$ in $\eta$ and depend on the factorization scale $\mu$. Such offdiagonal matrix elements are universal and appear not only in two-photon processes, but also in exclusive electroproduction of mesons $[46,47]$. They are (conformal) moments of the off-forward parton distributions introduced in [48,24-26]. Jain and Ralston pointed out that in QCD the first moment (given by the matrix element of a current) is related to elastic form factors [49]. A first non-perturbative calculation of the off-forward parton distributions in the bag model has been done recently [50].

Inserting the reduced expectation values (4.7) into Eq. (4.6) provides: 


$$
\begin{aligned}
T\left(\omega, \eta, Q^{2}\right)= & \int_{0}^{1} d u \int d^{n} x e^{i\{q x+\eta(u-1 / 2) P x\}} \\
& \times \sum_{k=0}^{\infty} \widetilde{C}_{k}\left(\frac{1}{x^{2}}\right)^{\left(2 l_{J}-l_{k}+k\right) / 2} \\
& \times(-i x P)^{k}[u(1-u)]^{\left(l_{k}+k\right) / 2-1} \\
& \times\left\langle P_{2}\left|O_{k}^{\mathrm{co}}(0)\right| P_{1}\right\rangle(\eta) .
\end{aligned}
$$

Employing the representation

$e^{i\{q x+\eta(u-1 / 2) P x\}}(-i)^{k}(x P)^{k}=(-\eta)^{-k} \frac{d^{k}}{d u^{k}} e^{i\{q x+\eta(u-1 / 2) P x\}}$

and after a Fourier transformation, using the definition of the hypergeometric functions

$$
\begin{aligned}
{ }_{2} F_{1}\left(\begin{array}{c}
\alpha, \beta \\
\gamma
\end{array} \mid x\right)= & \frac{1}{B(\alpha, \gamma-\alpha)} \\
& \times \int_{0}^{1} d u u^{\alpha-1}(1-u)^{\gamma-\alpha-1}(1-u x)^{-\beta},
\end{aligned}
$$

the desired result reads

$$
\begin{aligned}
T\left(\omega, \eta, Q^{2}\right)= & \sum_{k=0}^{\infty}\left(Q^{2}\right)^{-p} C_{k}\left(\eta \omega ; Q^{2} / \mu^{2}\right) \\
& \times\left(\frac{2 \omega}{1+\eta \omega}\right)^{k}\left\langle P_{2}\left|O_{k}^{\mathrm{co}}\left(\mu^{2}\right)\right| P_{1}\right\rangle(\eta),
\end{aligned}
$$

where the exponent of $Q^{2}$ is given by $p=\left(n+l_{k}^{\text {can }}-k\right.$ $\left.-2 l_{J}^{\text {can }}\right) / 2$. The $\mu$ dependence of the coefficients $C_{k}\left(\eta \omega ; Q^{2} / \mu^{2}\right)$ is governed by the renormalization group equation (3.46). They are known up to the normalization

$$
C_{k}(x ; 1)=c_{k}(1+x)^{-\left(n+l_{k}-k-2 l_{J}\right) / 2}{ }_{2} F_{1}\left(\begin{array}{c|c}
\left(k+l_{k}\right) / 2,\left(n+k+l_{k}-2 l_{J}\right) / 2 \\
k+l_{k}
\end{array} \mid \frac{2 x}{1+x}\right),
$$

where $l_{k}=2 d^{\text {can }}+k+\gamma_{k}$. From the properties of the hypergeometric functions, the symmetry relation $C_{k}(x)$ $=(-1)^{k} C_{k}(-x)$ follows. The overall normalization can be computed in the forward case $c_{k}=C_{k}(0)$. The reduced expectation values $\left\langle P_{2}\left|O_{k}^{\mathrm{co}}\left(\mu^{2}\right)\right| P_{1}\right\rangle(\eta)$ satisfy the diagonal renormalization group equation (3.47), which means that the $\eta$ dependence remains invariant under evolution and only the normalization will change.

\section{B. QCD predictions}

The conformally covariant OPE for two electromagnetic currents can now be applied in the same manner to predict different two-photon processes and their scattering amplitudes. In the conformal limit the same Wilson coefficients appear for quite different processes at leading twist-2. The process dependence comes from the non-perturbative expectation values of the conformal operators. For instance, the coefficient $\left(E_{k}^{i}\right) C_{k}^{i}$ appears in both polarized DIS and the two-photon production of (pseudo) scalar mesons at large momentum transfer as well as in the kinematical decomposition of the hadronic tensor for DVCS or for hadron production. Employing the conformal OPE (3.48) and performing steps analogous to those in the previous subsection leads to the prediction for the trace and the antisymmetric twist- 2 part (proportional to the $\epsilon$-tensor) of the different hadronic tensors in the conformal limit, which is, up to trivial kinematical factors, given by:

$$
\begin{aligned}
\left\{\begin{array}{c}
F\left(\omega, \eta, Q^{2}\right) \\
g_{1}\left(\omega, \eta, Q^{2}\right)
\end{array}\right\}= & \sum_{k=0}^{\infty} \sum_{i}\left\{\begin{array}{c}
{ }^{i} C_{k}\left(\eta \omega ; Q^{2} / \mu^{2}\right) \\
{ }^{i} E_{k}\left(\eta \omega ; Q^{2} / \mu^{2}\right)
\end{array}\right\}\left(\frac{2 \omega}{1+\eta \omega}\right)^{k} \\
& \times\left\{\begin{array}{c}
\left\langle P_{2}\left|{ }^{i} O_{k}^{\mathrm{co}}\left(\mu^{2}\right)\right| P_{1}\right\rangle(\eta) \\
\left\langle P_{2}\left|{ }^{i} R_{k}^{\mathrm{co}}\left(\mu^{2}\right)\right| P_{1}\right\rangle(\eta)
\end{array}\right\}
\end{aligned}
$$

where the coefficient functions read

$$
\begin{aligned}
\left\{\begin{array}{c}
{ }^{i} C_{k}(x ; 1) \\
{ }^{i} E_{k}(x ; 1)
\end{array}\right\}= & \left\{\begin{array}{c}
{ }^{i} c_{k} \\
{ }^{i} e_{k}
\end{array}\right\}(1+x)^{-1-{ }^{i} \gamma_{k} / 2}{ }_{2} F_{1} \\
& \times\left(\begin{array}{c}
k+1+\frac{1}{2}{ }^{i} \gamma_{k}, k+2+\frac{1}{2}{ }^{i} \gamma_{k} \\
2 k+4+{ }^{i} \gamma_{k}
\end{array} \mid \frac{2 x}{1+x}\right) .
\end{aligned}
$$

Without restrictions the obtained predictions are valid at LO. Thus, also the evolution equations for the off-diagonal parton distribution amplitudes are solved easily in terms of the conformal moments [44]. In NLO the correction due to the $\beta$-function appears originally only in the off-diagonal matrix elements of the anomalous-dimension matrices. From the NLO calculation of the pion evolution kernel [16-19] and also from the conformal constraints (3.17), this correction is known for the non-singlet channel. Beyond this order it is expected that also the coefficient functions will be offdiagonal, because of the conformal symmetry breaking proportional to the $\beta$-function. 
In the forward case, i.e. $\eta=0$, the conformal expansion is related to the moments in DIS by a dispersion relation:

$$
\left\{\begin{array}{c}
F\left(\omega, Q^{2}\right) \\
g_{1}\left(\omega, Q^{2}\right)
\end{array}\right\}=\sum_{k=0}^{\infty} \sum_{i} \omega^{k}\left\{\begin{array}{c}
{ }^{i} c_{k}\left\langle P\left|{ }^{i} O_{k}\left(\mu^{2}\right)\right| P\right\rangle \\
{ }^{i} e_{k}\left\langle\left. P\right|^{i} R_{k}\left(\mu^{2}\right) \mid P\right\rangle
\end{array}\right\}
$$

where the additional factor $2^{k}$ has been absorbed into the reduced expectation values [compare with Eq. (4.7)]. Thus, the overall normalization ${ }^{i} C_{k}(0 ; 1)={ }^{i} c_{k}$ and ${ }^{i} E_{k}(0 ; 1)={ }^{i} e_{k}$ can be taken from the calculations in unpolarized [51-53] and polarized [54] DIS, respectively, and are known up to order $\alpha_{s}^{2}$.

A further special case is $\eta=1$, where the conformal expansions give, up to a kinematical prefactor, the amplitudes for the production of pseudo-scalar and scalar mesons by virtual photons. Here complete NLO calculations were performed in the non-singlet channel and can now serve as a consistency check.

\section{RADIATIVE CORRECTIONS TO MESON TRANSITION FORM FACTORS}

\section{A. Transition form factors and conformally covariant OPE}

The photon-to-meson transition form factor, measured in $\gamma^{\star}\left(q_{1}\right) \gamma^{\star}\left(q_{2}\right) \rightarrow M(P)$, is given at large momentum transfer as a convolution of the hard-scattering amplitude $T\left(\omega, x, \alpha_{s}\right)$ and the meson distribution amplitude (DA) $\phi\left(x, Q^{2}\right)[55]$,

$$
\begin{gathered}
\Gamma_{\alpha \beta}=\epsilon_{\alpha \beta \mu \nu} q_{1}^{\mu} q_{2}^{\nu} F\left(\omega, Q^{2}\right), \\
F\left(\omega, Q^{2}\right)=\frac{N}{Q^{2}} T\left(\omega, x, \alpha_{s}\left(Q^{2}\right)\right) \otimes \phi\left(x, Q^{2}\right) .
\end{gathered}
$$

The kinematical variables are defined as before by $\omega$ $=P q / Q^{2}$ and $q=\left(q_{1}-q_{2}\right) / 2$. The factor $N$ is determined by the underlying flavor structure, e.g. for the $\pi^{0}$ meson $N$ $=e_{u}^{2}-e_{d}^{2}$. The hard-scattering amplitude is given perturbatively by

$$
\begin{aligned}
T\left(\omega, x, \alpha_{s}\right)= & \hat{T}^{(0)}(\omega, x)+\frac{\alpha_{s}}{2 \pi} \hat{T}^{(1)}(\omega, x) \\
& +O\left(\alpha_{s}^{2}\right)+\{x \rightarrow 1-x\}, \\
\hat{T}^{(0)}(\omega, x)= & \frac{1}{1+\omega[(1-x)-x]}, \\
V\left(x, y ; \alpha_{s}\right)= & \frac{\alpha_{s}}{2 \pi}\left[V^{(0)}(x, y)\right]_{+} \\
& +\left(\frac{\alpha_{s}}{2 \pi}\right)^{2}\left[V^{(1)}(x, y)\right]_{+}+O\left(\alpha_{s}^{3}\right),
\end{aligned}
$$

$$
\begin{aligned}
V^{(0)}(x, y)= & C_{F} \theta(y-x) \frac{x}{y}\left(1+\frac{1}{y-x}\right) \\
& +\left\{\begin{array}{l}
x \rightarrow 1-x \\
y \rightarrow 1-y
\end{array}\right\},
\end{aligned}
$$

where the + -prescription is defined as $[V(x, y)]_{+}=V(x, y)$ $-\delta(x-y) \int d z V(z, y)$.

The given formulas coincide at leading order with the prediction of the conformally covariant OPE (4.13) for $g_{1}\left(\omega, \eta=1, Q^{2}\right)$. To make this correspondence explicit, we expand the distribution amplitude in terms of the eigenfunctions of the evolution kernel, which is actually given by the conformal spin expansion

$$
\begin{aligned}
\phi\left(x, Q^{2}\right) & =\sum_{k=0}^{\infty} \frac{(1-x) x}{N_{k}} C_{k}^{3 / 2}(2 x-1)\left\langle P\left|O_{k k}\left(\mu^{2}\right)\right| 0\right\rangle_{\mid \mu^{2}=Q^{2}}^{r e d} \\
N_{k} & =\frac{(k+1)(k+2)}{4(2 k+3)}
\end{aligned}
$$

Taking into account the definition of the Gegenbauer polynomials

$$
\frac{(1-x) x}{N_{k}} C_{k}^{3 / 2}(2 x-1)=(-1)^{k} \frac{2(2 k+3)}{(k+1) !} \frac{d^{k}}{d x^{k}}[x(1-x)]^{k+1},
$$

the transition form factor (5.1) reads

$$
\begin{aligned}
F\left(\omega, Q^{2}\right)= & \frac{N}{Q^{2}} \sum_{k=0}^{\infty} \frac{2(2 k+3)}{(k+1) !} \int_{0}^{1} d x[x(1-x)]^{k+1} \\
& \times \frac{d^{k}}{d x^{k}}\left(\frac{1}{1+\omega[(1-x)-x]}+\frac{1}{1+\omega[x-(1-x)]}\right) \\
& \times\left\langle P\left|O_{k k}\left(\mu^{2}\right)\right| 0\right\rangle_{\mid \mu^{2}=Q^{2}}^{r e d}
\end{aligned}
$$

Performing the differentiation and using the definition of hypergeometric functions (4.10), Eq. (5.8) coincides-up to different normalization factors for the composite operatorswith the conformal OPE prediction (4.13) to LO:

$$
\begin{aligned}
F\left(\omega, Q^{2}\right)= & \frac{N}{Q^{2}} \sum_{k=0}^{\infty} B(k+1, k+2) \frac{2(2 \omega)^{k}}{(1+\omega)^{k+1}} \\
& \times{ }_{2} F_{1}\left(\begin{array}{c}
k+1, k+2 \\
2(k+2)
\end{array} \mid \frac{2 \omega}{1+\omega}\right) \\
& \times\left\langle P\left|O_{k k}\left(\mu^{2}\right)\right| 0\right\rangle_{\mid \mu^{2}=Q^{2}}^{\text {red }}+\{\omega \rightarrow-\omega\} .
\end{aligned}
$$

\section{B. Consistency check at next-to-leading order}

Now we are able to perform the consistency check of the existing NLO calculations. The $\alpha_{s}$ correction to the hard scattering part was computed by del Aguila and Chase [56] in the OPE approach and by Braaten [57] (these papers contain also the corrections to the scalar meson and the longitudinal component of the vector meson transition form factor, 
respectively) as well as by Radyushkin et al. [58] in the hard-scattering picture. The results are derived in the $\overline{\mathrm{MS}}$ scheme and the occurring $\gamma^{5}$ ambiguity in dimensional regularization was resolved with different methods. The results are in agreement. To show the structure most clearly, we rewrite their result in the following form:

$$
\begin{aligned}
\hat{T}^{(1)}(\omega, x)= & \hat{T}^{(0)}(\omega, z) \otimes\left[\left[V^{(0)}(z, x)\right]_{+} \ln \frac{Q^{2}}{\mu^{2}}\right. \\
& \left.-\frac{3}{2}\left[V^{\mathrm{b}}(z, x)\right]_{+}-\frac{3}{2} C_{F} \delta(z-x)\right] \\
& +\hat{T}^{(0)}(\omega, z) \ln \{1+\omega(\bar{z}-z)\} \otimes\left[V^{(0)}(z, x)\right]_{+} \\
& +\hat{T}^{(0)}(\omega, z) \otimes[g(z, x)]_{+}, \\
g(x, y)= & -C_{F} \theta(y-x) \frac{\ln \left(1-\frac{x}{y}\right)}{y-x}+\left\{\begin{array}{l}
x \rightarrow 1-x \\
y \rightarrow 1-y
\end{array}\right\}, \\
V^{\mathrm{b}}(x, y)= & C_{F} \theta(y-x) \frac{x}{y} \frac{1}{y-x}+\left\{\begin{array}{l}
x \rightarrow 1-x \\
y \rightarrow 1-y
\end{array}\right\},
\end{aligned}
$$

where only the renormalization scale was identified with the factorization scale $\mu$, so that latter remains explicit. The solution of the evolution equation in the conformal limit is known and given by the conformal spin expansion

$$
\phi\left(x, Q^{2}\right)=\sum_{k=0}^{\infty} \varphi_{k}^{\mathrm{ef}}\left(x, \alpha_{s}\right)\left\langle P\left|O_{k k}\left(\mu^{2}\right)\right| 0\right\rangle_{\mid \mu^{2}=Q^{2}}^{r e d},
$$

where the eigenfunctions of the evolution kernel can be written as

$$
\begin{aligned}
\varphi_{k}^{e f}\left(x, \alpha_{s}\right)= & (-1)^{k} \frac{2(3+2 k)}{(k+1) !} \frac{d^{k}}{d x^{k}} \\
& \times\left[x ^ { 1 + k } ( 1 - x ) ^ { 1 + k } \left(1+\frac{\alpha_{s}}{2 \pi} F_{k}(x)\right.\right. \\
& \left.\left.+O\left(\alpha_{s}^{2}\right)\right)\right]
\end{aligned}
$$

The $\alpha_{s}$ correction was obtained by the leading order calculation of the special conformal anomaly matrix for parityeven operators and employing the formula (3.25) for the transformation matrix $\hat{B}$ :

$$
\begin{aligned}
F_{k}(x)= & \left(\gamma_{k}^{(0)}+\beta_{0}\right)\left[\frac{1}{2} \ln (x(1-x))-\psi(2+k)+\psi(4+2 k)\right] \\
& +C_{F}\left[\frac{\ln ^{2}\left(\frac{1-x}{x}\right)}{2}-\sum_{i=1}^{1+k}\left(-\frac{1}{i}+\frac{1+\delta_{1 i}}{2+k}\right)\right. \\
& \times(\phi(1-x, 1, i)+\phi(x, 1, i)) \\
& +2\left(\frac{(3+2 k)(\psi(2+k)-\psi(1))}{(1+k)(2+k)}\right. \\
& \left.\left.+\psi^{\prime}(2+k)-\frac{\pi^{2}}{4}\right)\right]
\end{aligned}
$$

where $\psi^{\prime}(z)=d \psi(z) / d z$ and $\phi(x, 1, i)=\sum_{k=0}^{\infty} x^{k} /(i+k)$ are the Lerch transcendent. This result coincides with the calculated evolution kernel in NLO [16-19]. The authors used the naive $\overline{\mathrm{MS}}$ scheme in which $\gamma^{5}$ is anticommutative, implying that the evolution kernel for pseudo-scalar mesons is the same as for scalar ones. For convenience we rewrite the $\alpha_{s}$ correction as a convolution:

$$
\begin{aligned}
\varphi_{n}^{e f}\left(x, \alpha_{s}\right)= & \left(\delta(x-y)+\frac{\alpha_{s}}{2 \pi} c^{(1)}(x, y)+\cdots\right) \\
& \otimes \frac{(1-y) y}{N_{n}} C_{n}^{3 / 2}(2 y-1),
\end{aligned}
$$

where ${ }^{5}$

$$
\begin{aligned}
c^{(1)}(x, y)= & (I-\mathcal{P})\left(\frac{\beta_{0}}{2} S(x, y)-S(x, z) \otimes V^{(0)}(z, y)\right. \\
& \left.-[g(x, y)]_{+}\right) .
\end{aligned}
$$

Furthermore, the shift operator $S(x, y)$ is implicitly defined by

$$
\begin{aligned}
& S(x, y) \otimes \frac{(1-y) y}{N_{n}} C_{n}^{3 / 2}(2 y-1) \\
& =\frac{d}{d \rho} \frac{((1-x) x)^{1+\rho}}{N_{n}} C_{n}^{3 / 2+\rho}(2 x-1)_{\mid \rho=0},
\end{aligned}
$$

$I$ is the identity and the operator $\mathcal{P}$ projects onto the diagonal part of the expansion of a function $f(x, y)$ with respect to $C_{i}^{3 / 2}$; i.e. $\mathcal{P} f(x, y)=\sum_{i=0}^{\infty}(1-x) x / N_{i} C_{i}^{3 / 2}(2 x-1) f_{i i} C_{i}^{3 / 2}(2 y$ $-1)$, where $f_{i j}$ with $0 \leqslant i, j \leqslant \infty$ are the expansion coefficients.

\footnotetext{
${ }^{5}$ The definition of $c^{(1)}(x, y)$ in [22,59] contains a misprint concerning the sign of $g(x, y)$.
} 
The hard-scattering part and the evolution kernel were computed in the same scheme. The convolution of the hardscattering part (5.10) with the solution (5.14) for $\beta=0$ yields:

$$
\begin{aligned}
F= & \frac{N}{Q^{2}} \sum_{k=0}^{\infty}\left(T^{(0)}+\frac{\alpha_{s}}{2 \pi} \mathcal{T}^{(1)}\right) \otimes \frac{(1-x) x}{N_{k}} \\
& \times C_{k}^{3 / 2}(2 x-1)\left\langle P\left|O_{k k}\right| 0\right\rangle^{r e d} .
\end{aligned}
$$

The off-diagonal part of $g(x, y)$ in the hard-scattering amplitude and the eigenfunctions cancel with each other and only the diagonal part is left. After decomposition of the term $\hat{T}^{(0)}(\omega, z) \ln \{1+\omega(\bar{z}-z)\}$ into a diagonal and an off-diagonal part $^{6}$ the $\alpha_{s}$ correction to the hard-scattering amplitude reads symbolically

$$
\begin{aligned}
\hat{\mathcal{T}}^{(1)}(\omega, x)= & \hat{T}^{(0)}(\omega, z) \otimes\left[\left[V^{(0)}(z, x)\right]_{+} \ln \frac{Q^{2}}{\mu^{2}}\right. \\
& \left.-\frac{3}{2}\left[V^{\mathrm{b}}(z, x)\right]_{+}-\frac{3}{2} C_{F} \delta(z-x)+\mathcal{P}[g(z, x)]_{+}\right] \\
& +\mathcal{P} \hat{T}^{(0)}(\omega, z) \ln \{1+\omega(\bar{z}-z)\} \otimes\left[V^{(0)}(z, x)\right]_{+} \\
& +(I-\mathcal{P})\left[\hat{T}^{(0)}(\omega, z) \ln \{1+\omega(\bar{z}-z)\}\right. \\
& \left.-\hat{T}^{(0)}(\omega, y) \otimes S(y, z)\right] \otimes\left[V^{(0)}(z, x)\right]_{+} .
\end{aligned}
$$

The first two lines contain only diagonal terms, which provide the $\alpha_{s}$ corrections to the overall normalization of the Wilson coefficients for the conformally covariant OPE. The off-diagonal terms in the last line generate the shift of the canonical dimension by the anomalous one. This can be seen by a straightforward calculation:

$$
\begin{gathered}
(I-\mathcal{P})\left[\hat{T}^{(0)} \ln \{1+\omega(\bar{z}-z)\}-\hat{T}^{(0)} \otimes S\right] \otimes\left[V^{(0)}\right]_{+} \otimes \frac{(1-x) x}{N_{k}} C_{k}^{3 / 2}(2 x-1) \\
\quad=\frac{\gamma_{k}^{(0)}}{2} \frac{(2 \omega)^{k}}{(1+\omega)^{k+1}} \frac{d}{d \rho}(1+\omega)^{-\rho_{2} F_{1}}\left(\begin{array}{c}
k+1+\rho, k+2+\rho \mid \\
2(k+2+\rho)
\end{array} \mid \frac{2 \omega}{1+\omega}\right)_{\mid \rho=0} .
\end{gathered}
$$

Therefore, this term coincides with the conformal prediction for the structure of the Wilson coefficients (4.14).

It remains to be shown that the normalization is consistent with the NLO calculation of the non-singlet sector for the polarized structure function $g_{1}$ measured in DIS. It is known that the diagonal part of the pion evolution kernel coincides with the non-singlet splitting kernel. This was analytically shown in $[48,23]$ by taking the limit of an extended pion evolution kernel:

$$
P(z)=\lim _{\eta \rightarrow 0} \frac{1}{|2 \eta|} \gamma\left(\frac{z}{\eta}, \frac{1}{\eta}\right), \quad \gamma\left(t, t^{\prime}\right)=V\left(\frac{1+t}{2}, \frac{1+t^{\prime}}{2}\right)_{\mid \theta\left(t-t^{\prime}\right) \rightarrow \epsilon(1-t) \theta\left[(1-t) /\left(1-t^{\prime}\right)\right] \theta\left[\left(t-t^{\prime}\right) /\left(1-t^{\prime}\right)\right]} .
$$

The extension of $V\left(t, t^{\prime}\right)$ into the whole $t, t^{\prime}$-plane is unique and it is done in practice by replacing the corresponding $\theta$ functions.

As mentioned before, the off-diagonal part in Eq. (5.17) does not contribute to the forward case. For the terms of the diagonal part that are given as a convolution with $T^{(0)}$ the procedure (5.19) provides the following NLO corrections:

$$
\begin{array}{r}
C_{F} \int_{0}^{1} d x\left(\left[\frac{1+x^{2}}{1-x}\right]_{+} \ln \frac{Q^{2}}{\mu^{2}}-\frac{3}{2}\left[\frac{2 x}{1-x}\right]_{+}\right. \\
\left.-\frac{3}{2} \delta(1-x)-2\left[\frac{\ln (1-x)}{1-x}\right]_{+}\right) x^{k} .
\end{array}
$$

\footnotetext{
${ }^{6}$ Diagonal refers to terms that contribute only to the normalization of the partial waves in the expansion (5.9), while off-diagonal terms cannot be represented in such an expansion with the given hypergeometric functions.
}

Convoluting the remaining term, $\mathcal{P} \hat{T}^{(0)}(\omega, z) \ln \{1+\omega(\bar{z}-z)\}$ $\otimes\left[V^{(0)}(z, x)\right]_{+}$, first with the Gegenbauer polynomials and then extracting the diagonal part gives:

$$
\begin{gathered}
-C_{F}[\psi(k+1)-\psi(1)]\left[\frac{3}{2}+\frac{1}{(k+1)(k+2)}\right. \\
-2 \psi(k+2)+2 \psi(1)] .
\end{gathered}
$$

Putting Eqs. (5.20) and (5.21) together, the whole NLO contribution to the overall normalization follows:

$$
\begin{aligned}
e_{k}= & 1+\frac{\alpha_{s}}{2 \pi} C_{F}\left(\operatorname { l n } \frac { Q ^ { 2 } } { \mu ^ { 2 } } \left[\frac{3}{2}+\frac{1}{(k+1)(k+2)}\right.\right. \\
& \left.-2 S_{1}(k+1)\right]+2 S_{1,1}(k)-2 S_{2}(k)+\left[\frac{1}{k+1}+\frac{1}{k+2}\right. \\
& \left.\left.+\frac{3}{2}\right] S_{1}(k)+\frac{3}{k+1}-\frac{9}{2}\right)
\end{aligned}
$$


where $S_{m}(k)=\sum_{i=1}^{k}(1 / i)^{m}$ and $S_{m, n}(k)=\sum_{i=1}^{k}(1 / i)^{m} S_{n}(i)$. Taking into account the different definition of moments in DIS, i.e. $k \rightarrow k-1$, the obtained normalization (5.22) coincides with the Wilson coefficients in longitudinal polarized DIS computed in Ref. [54] in the 't Hooft-VeltmanBreitenlohner-Maison (HVBM) scheme ${ }^{7}[60,61]$.

Finally, we show that also the NLO calculation for the transverse helicity amplitude $T_{++}$in the c.m. frame of the transition form factor for the non-singlet scalar mesons [56] coincides with the NLO corrections to unpolarized DIS. It is sufficient to consider the difference to the pseudo-scalar case, which can be written as a convolution of the hard scattering part with a diagonal kernel [56]:

$\hat{T}^{(0)}(\omega, z) \otimes \frac{\alpha_{s}}{2 \pi}\left(\left[V^{(0)}(z, x)\right]_{+}-\left[V^{\mathrm{b}}(z, x)\right]_{+}-\frac{C_{F}}{2} \delta(z-x)\right)$,

which has the eigenvalues $\left(\alpha_{s} / 2 \pi\right) C_{F} /((k+1)(k+2))$. Thus, the only difference to the pseudo-scalar case appears in the normalization given by these eigenvalues. In the notation of the DIS hadronic tensor, the considered helicity amplitude corresponds to the generalization of $F_{1}$ to non-forward processes. The difference of the corresponding DIS Wilson coefficient [51-53] and $e_{k}$ in Eq. (5.22) is precisely $\left(\alpha_{s} / 2 \pi\right) C_{F} /((k+1)(k+2))$ in NLO.

\section{A first view beyond NLO}

It has been shown to LO that the measured pion transition form factor at large momentum transfer [29,30], where one photon is almost real, supports the asymptotic distribution amplitude or even more narrow ones [62-64]. So it is phenomenologically very interesting to study the higher-order corrections to this distribution amplitude. In LO the asymptotic distribution amplitude $\varphi^{\text {as }}(x)=6 x(1-x)$ does not evolve with $Q^{2}$, but it is well known that this property is spoiled in the $\overline{\mathrm{MS}}$ scheme to NLO [65,59] by the mixing of the operators. In the conformal limit of the theory the conformal normalization conditions restore the non-evolution of the asymptotic distribution amplitude. The pion transition form factor for this amplitude is given by the first term of the conformal OPE (4.11):

$$
Q^{2} F\left(\omega, Q^{2}\right)=\frac{\sqrt{2} f_{\pi}}{3} \frac{2}{1+\omega^{2}} F_{1}\left(\begin{array}{c|c}
1,2 & 2 \omega \\
4 & \frac{2 \omega}{1+\omega}
\end{array}\right) c_{0}\left(\alpha_{s}\right),
$$

where the expectation value of the first operator, given by the axial current, provided the pion decay constant $f_{\pi}$

\footnotetext{
${ }^{7}$ In the HVBM scheme the anticommutativity of $\gamma^{5}$ in the nonsinglet sector is restored effectively by a finite renormalization. In this case, it is equivalent to the naive $\gamma^{5}$ prescription, which was used for the calculation of the NLO correction to the pion transition form factor.
}

$=130.7 \mathrm{MeV}$. The coefficient $c_{0}\left(\alpha_{s}\right)$ is normalized to 1 at LO. For the case that one photon is almost real, i.e. $\omega=1$, we get

$$
Q^{2} F\left(1, Q^{2}\right)=\sqrt{2} f_{\pi} c_{0}\left(\alpha_{s}\right)=0.185 c_{0}\left(\alpha_{s}\right) \mathrm{GeV}
$$

The predictive power of the conformal OPE tells us that the coefficient $c_{0}\left(\alpha_{s}\right)$ is the value of the Bjorken sum rule, which is calculated up to order $\alpha_{s}^{3}[66,3]$. For three active flavors the numerical result reads ${ }^{8}$

$$
c_{0}\left(\alpha_{s}\right)=1-\frac{\alpha_{s}}{\pi}-3.58333\left(\frac{\alpha_{s}}{\pi}\right)^{2}-20.21527\left(\frac{\alpha_{s}}{\pi}\right)^{3}+O\left(\alpha_{s}^{4}\right)
$$

Now we can give a rough estimate of the higher-loop corrections, which reduce the $\mathrm{LO}$ prediction at a scale of $Q^{2}$ $=2 \mathrm{GeV}^{2}$, where $\alpha_{s}$ is assumed to be 0.35 , by about $18 \%$, coinciding very well with the experimental results at this scale $[29,30]$. Note that the $\alpha_{s}^{2}$ correction to the coefficient function of $g_{1}$ is given in Ref. [54] and, therefore, the nextto-next-to-leading order (NNLO) prediction for the photonto-pion transition form factor can be also given in the conformal limit for arbitrary DA's.

Now let us consider the effects coming from the conformal symmetry breaking, which is manifested in the offdiagonal part of the Wilson coefficients and the anomalous dimension matrix. In the conformally covariant subtraction scheme considered here these terms are induced by the renormalization of the coupling and have to be proportional to the $\beta$-function. In NLO only the first coefficient of $\beta / g$ $=-\alpha_{s} /(4 \pi) \beta_{0}+O\left(\alpha_{s}^{2}\right)$ with $\beta_{0}=11-2 n_{f} / 3$ enters in the anomalous dimension matrix (or in the evolution kernel) and the off-diagonal term related to it was correctly predicted by the conformal constraints (3.17). On the other hand it is obvious that in this order, this off-diagonal term can be simply calculated from the two-loop diagram contributing the $n_{f}$-dependent part to the gluon vacuum polarization (quark bubble) $[69,70]$. In NNLO terms proportional to the $\beta$-function appear in both the Wilson coefficients and the evolution kernel. While the off-diagonal part to the Wilson coefficients can be obtained in the same manner as described, the special conformal anomaly should be known in order $\alpha_{s}^{2}$ to treat the evolution of the DA in the correctly.

Although, the $n_{f}$-dependent part of the Wilson coefficients in NNLO can be obtained from the result given in $[70,71]$, we only discuss here the conformal symmetry breaking in NLO. Because of this breaking the asymptotic distribution amplitude will evolve also in the conformal subtraction scheme. Thus, let us first study the fixed $\alpha_{s}$ regime, where a term proportional to $\beta_{0}$ arises in the $\alpha_{s}$ corrections to the eigenfunctions (5.13) responsible for the evolution of the asymptotic distribution amplitude. A renormalization group transformation absorbs this term into the hard scattering part:

\footnotetext{
${ }^{8}$ The $\alpha_{s}^{4}$ correction has been estimated to be negative too $[67,68]$.
} 


$$
c_{0}^{\mathrm{fix}}\left(\alpha_{s}\right)=1-\frac{\alpha_{s}}{2 \pi}\left(2+\frac{\beta_{0}}{6}\right)+O\left(\alpha_{s}^{2}\right) .
$$

Note that this $\beta_{0}$ term was predicted from the $\beta$-function by the conformal constraint (3.17) and represents the first term of the series $\beta / g\left(1 / 3+O\left(\alpha_{s}\right)\right)$. For the above values the $\beta_{0}$ term provides a reduction of about $8 \%$, so that the whole net reduction in NLO is of about $19.5 \%$. Because of a partial cancellation between the different conformal symmetry breaking terms [59] for the lowest moments, this reduction is similar to that in the $\overline{\mathrm{MS}}$ scheme, where the correction to the hard scattering amplitude is $1-5 \alpha_{s} /(3 \pi)$.

Now we consider the real case, where the coupling constant is running. Then, the evolution of the asymptotic distribution amplitude is only avoidable if the matrix $\hat{B}\left(\alpha_{s}\right)$, which diagonalizes the anomalous dimension matrix is renormalization-group-invariant, i.e. $\hat{B}\left(\alpha_{s}(\mu), \mu\right)$ depends explicitly on $\mu$. Here we proceed in the manner proposed in $[20,65]$, which was explored in more detail in [59]. Note that already a renormalization group transformation was done to diagonalize the kernel for fixed $\alpha_{s}$, so that the evolution kernel is different from that in the $\overline{\mathrm{MS}}$ scheme. Generally, the non-perturbative input can be taken from sum rules, lattice or model calculations at a lower scale. Assuming that the "input" $6 x(1-x)$ is given at a scale of $Q \sim 1 \mathrm{GeV}$ the evolution provides an additional negative effect of almost $2 \%$ for $Q^{2}=2 \mathrm{GeV}^{2}$ and of almost $3.5 \%$ for $Q^{2}$ $=8 \mathrm{GeV}^{2}$. The resulting prediction is 0.148 at $Q^{2}$ $=2 \mathrm{GeV}^{2}$. A more detailed analysis including other distribution amplitudes will be given elsewhere.

\section{CONCLUSION}

In this article we reviewed an appropriate technique, developed previously, based on the true conformal Ward identities and conformal constraints, to analyze conformal symmetry breaking in a massless quantum field theory due to the renormalization of the UV-divergences. Since we are dealing with Ward identities for the basis fields to define the anomalous terms of gauge-invariant operators the conformal symmetry is also spoiled by the gauge fixing. However, finally we are interested in predicting physical quantities from which these terms should be absent. This point of view allows us to understand conformal symmetry and its breaking in quantum field theories without further conformal assumptions that led in the past to conflicts between conformal predictions and explicit calculations. This approach is also sufficient to reanalyze more directly the failure of the conformal prediction from the light-cone expansion of a non-local operator for the eigenfunctions of the pion evolution kernel in gauge field theory.

We employed this technique to prove that a factorization scheme exists in which the conformal covariance of composite operators holds true in the conformal limit of the theory. The transformation from an arbitrary scheme to the conformal scheme is given by the special-conformal anomaly matrix. Consequently, the essential assumption to construct the conformally covariant OPE of two local currents can be ful- filled by requiring appropriate normalization conditions. This OPE provides powerful scheme-independent predictions, which were used for exclusive two-photon processes in the generalized Bjorken region; also, restricted to the conformal limit, for simplicity we did not consider the full kinematical structure of the hadronic tensor. Since these predictions are scheme-independent they hold true in any scheme; however, it is not a trivial task to see this in the explicit calculated expressions beyond the LO.

At this stage it is not clear how to obtain, in an economical way, the terms proportional to the $\beta$-function that are missing in the conformal limit. It seems to be worthwhile studying if the non-decomposable irreducible representations that allow us to include the $\beta$-function in the conformal symmetry interpretation also have predictive power. This would avoid having to formally rely on the hypothetical conformal limit. A second point of view is to use the common irreducible representations and to consider the conformal symmetrybreaking terms as perturbation proportional to the $\beta$-function. It is very interesting that in the case of the Crewther relation the $\beta$-function can be absorbed by the BLM scale-fixing prescription into the scale of the coupling constant $[7,72,8]$.

In NLO conformal symmetry-breaking terms do not appear in the Wilson coefficients. The $\beta$-dependence of the anomalous-dimension matrix is predicted by the conformal constraints for the dimensional regularized theory in the $\overline{\mathrm{MS}}$ scheme; however, the renormalization group transformation to the conformal scheme provides an additional $\beta$-dependence of the anomalous-dimension matrix in terms of the special-conformal anomaly matrix computed in the $\overline{\mathrm{MS}}$ scheme. This can be applied in a straightforward manner to predict the evolution in the singlet channel to NLO only by a one-loop calculation of the special-conformal anomaly matrix.

\section{ACKNOWLEDGMENTS}

I would like to thank S. Brodsky, R. Crewther and A.L. Kataev for discussions, which inspired me to study the conformal properties of the OPE in QCD. The author is grateful to the CERN Theory Division for its hospitality during his visit, where this work was done. He was financially supported by the Deutsche Forschungsgemeinschaft (DFG).

\section{APPENDIX}

Here we carry out the calculation providing Eq. (3.30). For this reason, we first show the identity:

$$
\begin{gathered}
{[\hat{a}, \hat{B}]_{k k^{\prime}}=a\left(k, k^{\prime}\right) B_{k k^{\prime}}=-\left\{\hat{\gamma}^{c}\left(k^{\prime}\right) \hat{B}\right\}_{k k^{\prime}},} \\
a\left(k, k^{\prime}\right)=2\left(k-k^{\prime}\right)\left(k+k^{\prime}+\nu_{1}+\nu_{2}\right) .
\end{gathered}
$$

Let us mention that by iteration and taking into account $B_{k k}=1$, Eq. (3.25) follows. In accordance with the definition (3.23) of the matrix $\hat{B}$ we introduce the notation 


$$
\begin{gathered}
B_{k k^{\prime}}=\sum_{i=0}^{\infty}\left\{\hat{\Gamma}\left(k^{\prime}\right)^{i}\right\}_{k k^{\prime}}, \quad \hat{\Gamma}^{0}=\hat{1}, \\
\text { and } \Gamma_{m n}\left(k^{\prime}\right)=\mathcal{L}_{k^{\prime}} \gamma_{m n}^{\mathrm{ND}}=-\frac{\gamma_{m n}^{\mathrm{ND}}}{\gamma_{m}-\gamma_{k^{\prime}}}
\end{gathered}
$$

as well as the inverse operator $\mathcal{L}_{k^{\prime}}^{-1}$ : $\mathcal{L}_{k^{\prime}}^{-1} \gamma_{m n}^{\mathrm{ND}}=-\left(\gamma_{m}-\gamma_{k^{\prime}}\right) \gamma_{m n}^{\mathrm{ND}}$. From the conformal constraints (3.18) we obtain formally:

$$
\left[\hat{a}, \hat{\Gamma}\left(k^{\prime}\right)\right]=-\hat{\gamma}^{c}(l)+\mathcal{L}_{k^{\prime}} \hat{K}(l)+\mathcal{L}_{k^{\prime}} \hat{\gamma}^{c}(l) \mathcal{L}_{k^{\prime}}^{-1},
$$

where $\hat{K}(l)=\left[\hat{\gamma}^{\mathrm{ND}}, \hat{\gamma}^{c}(l)\right]$. Note that the $l$-independence of the RHS is ensured by the constraints (3.28) and that the last term on this side induces for $\left[\hat{a}, \hat{\Gamma}\left(k^{\prime}\right)\right]_{k k^{\prime}}$ the contribution $\left\{\mathcal{L}_{k^{\prime}} \hat{\gamma}^{c}(l) \mathcal{L}_{k^{\prime}}^{-1} \hat{1}\right\}_{k k^{\prime}}=\hat{\gamma}_{k k^{\prime}}^{c}(l) \delta_{k k^{\prime}}$, so that $\left[\hat{a}, \hat{\Gamma}\left(k^{\prime}\right)\right]_{k^{\prime} k^{\prime}}=0$ is identically satisfied for $k=k^{\prime}$. Repeated application of

$$
\begin{aligned}
{\left[\hat{a}, \hat{\Gamma}\left(k^{\prime}\right)^{i-1} \hat{\Gamma}\left(k^{\prime}\right)\right]=} & {\left[\hat{a}, \hat{\Gamma}\left(k^{\prime}\right)^{i-1}\right] \hat{\Gamma}\left(k^{\prime}\right) } \\
& +\hat{\Gamma}\left(k^{\prime}\right)^{i-1}\left[\hat{a}, \hat{\Gamma}\left(k^{\prime}\right)\right]
\end{aligned}
$$

then implies the following form:

$$
\begin{aligned}
{\left[\hat{a}, \hat{\Gamma}\left(k^{\prime}\right)^{i}\right]=} & -\hat{\gamma}^{c}(l) \hat{\Gamma}\left(k^{\prime}\right)^{i-1}-\hat{R}^{(i-1)}\left(k^{\prime}, l\right) \\
& +\hat{R}^{(i)}\left(k^{\prime}, l\right)+\hat{\Gamma}\left(k^{\prime}\right)^{i-1} \mathcal{L}_{k^{\prime}} \hat{\gamma}^{c}(l) \mathcal{L}_{k^{\prime}}^{-1},
\end{aligned}
$$

with $\hat{R}^{(-1)}=0$. For $\left[\hat{a}, \hat{\Gamma}\left(k^{\prime}\right)^{i}\right]_{k k^{\prime}}$ the last term in the RHS is proportional to $\gamma^{c}(l)_{k^{\prime} k^{\prime}}$ and, because of the property

[1] R. Crewther, Phys. Rev. Lett. 28, 1421 (1972).

[2] S. Adler, C. Callan, D. Gross, and R. Jackiw, Phys. Rev. D 6, 2982 (1972).

[3] S. Larin and J. Vermaseren, Phys. Lett. B 259, 345 (1991).

[4] S. Gorishny, A. Kataev, and S. Larin, Phys. Lett. B 259, 144 (1991).

[5] R. Crewther, Phys. Lett. B 397, 137 (1997).

[6] D. Broadhurst and A. Kataev, Phys. Lett. B 315, 179 (1993).

[7] H. Lu and S. Brodsky, Phys. Rev. D 48, 3310 (1995).

[8] J. Rathsman, Phys. Rev. D 54, 3420 (1996).

[9] S. Ferrara, R. Gatto, and A. Grillo, Phys. Lett. 36B, 124 (1971); 38B, 188(E) (1972).

[10] S. Ferrara, R. Gatto, and A. Grillo, Nucl. Phys. B34, 349 (1971)

[11] S. Ferrara, R. Gatto, and A. Grillo, Phys. Rev. D 5, 3102 (1972).

[12] L. Bonora, G. Sartori, and N. Tonin, Nuovo Cimento A 10, 667 (1972).

[13] N. Craigie, V. Dobrev, and I. Todorov, Ann. Phys. (N.Y.) 159, 411 (1985).

[14] S. Ferrara, A. Grillo, and G. Parisi, Nucl. Phys. B54, 552 (1973) $\gamma^{c}\left(k^{\prime}\right)_{k^{\prime} k^{\prime}}=0$, it can be avoided for $l=k^{\prime}$. Obviously, employing Eq. (A4) we get the identity (A1):

$$
\begin{aligned}
{[\hat{a}, \hat{B}]_{k k^{\prime}} } & =\sum_{i=0}^{\infty}\left[\hat{a}, \Gamma\left(k^{\prime}\right)^{i}\right]_{k k^{\prime}} \\
& =-\sum_{i=1}^{\infty}\left(\left\{\hat{\gamma}^{c}\left(k^{\prime}\right) \hat{\Gamma}\left(k^{\prime}\right)^{i-1}\right\}_{k k^{\prime}}+\hat{R}_{k k^{\prime}}^{(i-1)}-\hat{R}_{k k^{\prime}}^{(i)}\right) \\
& =-\sum_{i=1}^{\infty}\left\{\hat{\gamma}^{c}\left(k^{\prime}\right) \hat{\Gamma}\left(k^{\prime}\right)^{i-1}\right\}_{k k^{\prime}} \\
& =-\left\{\hat{\gamma}^{c}\left(k^{\prime}\right) \hat{B}\right\}_{k k^{\prime}} .
\end{aligned}
$$

With the help of relation (A1) the desired calculation is easy:

$$
\begin{aligned}
\left\{\hat{B}^{-1}\left[\hat{a}(l)+\hat{\gamma}^{c}(l)\right] \hat{B}\right\}_{k k^{\prime}}= & \left\{\hat{B}^{-1} \hat{B}\right\}_{k k^{\prime}} a\left(k^{\prime}, l\right)+\left\{\hat{B}^{-1}[\hat{a}, \hat{B}]\right. \\
& \left.+\hat{B}^{-1} \hat{\gamma}^{c}(l) \hat{B}\right\}_{k k^{\prime}} \\
= & a(k, l) \delta_{k k^{\prime}}+\left\{\hat { B } ^ { - 1 } \left[\hat{\gamma}^{c}(l)\right.\right. \\
& \left.\left.-\hat{\gamma}^{c}\left(k^{\prime}\right)\right] \hat{B}\right\}_{k k^{\prime}} \\
= & a(k, l) \delta_{k k^{\prime}}+2\left(k^{\prime}-l\right) \\
& \times\left\{\hat{B}^{-1} \hat{\gamma} \hat{B}\right\}_{k k^{\prime}},
\end{aligned}
$$

where we used $\hat{\gamma}^{c}(l)-\hat{\gamma}^{c}\left(k^{\prime}\right)=-2\left(l-k^{\prime}\right) \hat{\gamma}$. Since the matrix $\hat{B}$ diagonalizes the anomalous-dimension matrix the last line is identical with Eq. (3.30).
[15] S. Brodsky, Y. Frishman, G. Lepage, and C. Sachradja, Phys. Lett. 91B, 239 (1980).

[16] F.-M. Dittes and A. Radyushkin, Phys. Lett. 134B, 359 (1984).

[17] M. Sarmadi, Phys. Lett. 143B, 471 (1984).

[18] G. Katz, Phys. Rev. D 31, 652 (1985).

[19] S. Mikhailov and A. Radyushkin, Nucl. Phys. B254, 89 (1985).

[20] S. Brodsky, P. Damgaard, Y. Frishman, and G. Lepage, Phys. Rev. D 33, 1881 (1986).

[21] D. Müller, Z. Phys. C 49, 293 (1991).

[22] D. Müller, Phys. Rev. D 49, 2525 (1994).

[23] D. Müller et al., Fortschr. Phys. 42, 101 (1994).

[24] X. Ji, Phys. Rev. Lett. 78, 610 (1997).

[25] X. Ji, Phys. Rev. D 55, 7114 (1997).

[26] A. Radyushkin, Phys. Lett. B 380, 417 (1996).

[27] PLUTO Collaboration, C. Berger et al., Phys. Lett. 142B, 125 (1984).

[28] TPC/Two Gamma Collaboration, H. Aihara et al., Phys. Rev. Lett. 64, 172 (1990).

[29] CELLO Collaboration, H.-J. Behrend et al., Z. Phys. C 49, 401 (1991) 
[30] CLEO Collaboration, J. Gronberg et al., Phys. Rev. D 57, 33 (1998).

[31] G. Mack and A. Salam, Ann. Phys. (N.Y.) 53, 174 (1969).

[32] Y. Makeenko, Sov. J. Nucl. Phys. 33, 440 (1981).

[33] T. Ohrndorf, Nucl. Phys. B198, 26 (1982).

[34] I. Balitsky and V. Braun, Nucl. Phys. B311, 541 (1989).

[35] R. Zaikov, Lett. Math. Phys. 16, 1 (1988).

[36] G. Parisi, Phys. Lett. 39B, 643 (1972).

[37] N. Nielsen, Nucl. Phys. B65, 413 (1973).

[38] S. Sarkar, Nucl. Phys. B83, 108 (1974).

[39] N. Nielsen, Nucl. Phys. B120, 212 (1977).

[40] S. Adler, J. Collins, and A. Duncan, Phys. Rev. D 15, 1712 (1977).

[41] J. Collins, A. Duncan, and S. Joglekar, Phys. Rev. D 16, 438 (1977).

[42] P. Minkowski, Berne report 76-0813 (unpublished).

[43] M. Chase, Nucl. Phys. B167, 125 (1980).

[44] M. Chase, Nucl. Phys. B174, 109 (1980).

[45] B. Geyer, D. Müller, and D. Robaschik, in Proceedings of the 1992 Zeuthen Workshop on Elementary Particle Theory: "Deep Inelastic Scattering," edited by J. Blümlein and T. Riemann (Teupitz/Brandenburg, Germany, 1992) [Nucl. Phys. B (Proc. Suppl.) 29A, 22 (1992)].

[46] J. Collins, L. Frankfurt, and M. Strikman, Phys. Rev. D 56, 2982 (1997).

[47] A. Radyushkin, Phys. Lett. B 385, 333 (1996).

[48] F.-M. Dittes et al., Phys. Lett. B 209, 325 (1988).

[49] P. Jain and J. Ralston, Proceedings of the Workshop on Future Directions in Particle and Nuclear Physics at Multi-GeV Hadron Beam Facilities (Brookhaven National Laboratory, Upton, NY, 1993).
[50] X. Ji, W. Melnitchouk, and X. Song, Phys. Rev. D 56, 5511 (1997).

[51] W. V. Neerven and E. Zijlstra, Phys. Lett. B 272, 127 (1991).

[52] E. Zijlstra and W. van Neerven, Phys. Lett. B 273, 476 (1991).

[53] E. Zijlstra and W. van Neerven, Nucl. Phys. B383, 525 (1992).

[54] E. Zijlstra and W. van Neerven, Nucl. Phys. B417, 61 (1994); B426, 245(E) (1994).

[55] G. Lepage and S. Brodsky, Phys. Rev. D 22, 2157 (1980).

[56] F. D. Aguila and M. Chase, Nucl. Phys. B193, 517 (1981).

[57] E. Braaten, Phys. Rev. D 28, 524 (1983).

[58] E. Kadantseva, S. Mikhailov, and A. Radyushkin, Sov. J. Nucl. Phys. 44, 326 (1986).

[59] D. Müller, Phys. Rev. D 51, 3855 (1995).

[60] G. 't Hooft and M. Veltman, Nucl. Phys. B44, 189 (1972).

[61] P. Breitenlohner and D. Maison, Commun. Math. Phys. 52, 11 (1977).

[62] A. Radyushkin, CEBAF-TH-94-15 (unpublished).

[63] R. Jakob, P. Kroll, and M. Raulfs, J. Phys. G 22, 45 (1996).

[64] S. Ong, Phys. Rev. D 52, 3111 (1995).

[65] S. Mikhailov and A. Radyushkin, Nucl. Phys. B273, 297 (1986).

[66] S. Gorishny and S. Larin, Phys. Lett. B 172, 109 (1986).

[67] A. Kataev and V. Starstenko, Mod. Phys. Lett. A 10, 235 (1995).

[68] M. Samuel, J. Ellis, and M. Karliner, Phys. Rev. Lett. 74, 4380 (1995).

[69] S. Mikhailov, Phys. Lett. B 416, 421 (1997).

[70] P. Gosdzinsky and N. Kivel, hep-ph/9707367.

[71] A. Belitsky and A. Schäfer, hep-ph/9801252.

[72] S. Brodsky, G. Gabadadze, A. Kataev, and H. Lu, Phys. Lett. B 372, 133 (1996). 